\title{
EVENT GENERATOR FOR RHIC SPIN PHYSICS
}

September 21-23, 1998

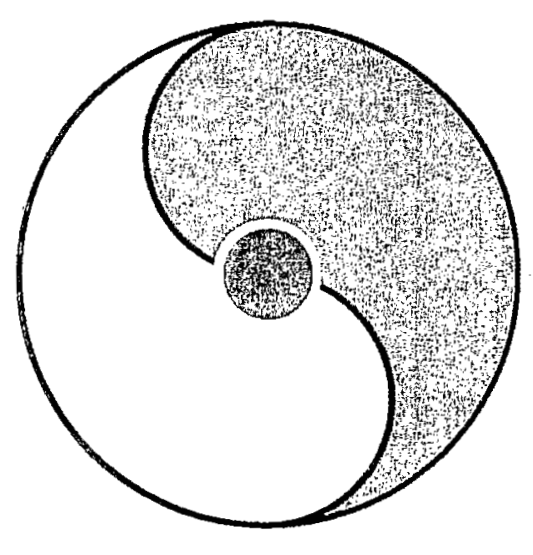

Organizers

Naohito Saito and Andreas Schaefer

RIKEN BNL Research Center

Building 510, Brookhaven National Laboratory, Upton, NY 11973, USA 


\section{Other RIKEN BNL Research Center Proceedings Volumes:}

Volume 10 - Physics of Polarimetry at RHIC - BNLAugust 4-7, 1998 - Organizers: Ken Imai and Doug Fields

Volume 9 - High Density Matter in AGS, SPS and RHIC Collisions - BNL-65762 July 11, 1998 - Organizers: Klaus Kinder-Geiger and Yang Pang

Volume 8 - Fermion Frontiers in Vector Lattice Gauge Theories - BNL-65634 May 6-9, 1998 - Organizers: Robert Mawhinney and Shigemi Ohta

Volume 7 - RHIC Spin Physics - BNL-65615 April 27-29, 1998 - Organizers: Gerry Bunce, Yousef Makdisi, Naohito Saito, Mike Tannenbaum and Aki Yokosawa

Volume 6 - Quarks and Gluons in the Nucleon - BNL-65234 November 28-29, 1997, Saitama, Japan Organizers: Tashiaki Shibata and Koichi Yazaki

Volume 5 - Color Superconductivity, Instantons and Parity (Non?)-Conservation at High Baryon Density - BNL-65105

November 11, 1997 - Organizer - Miklos Gyulassy

Volume 4 - Inauguration Ceremony, September 22 and Non-Equilibrium Many Body Dynamics - BNL- 64912 September 23-25, ${ }^{\circ} 1997$ - Organizer - Miklos Gyulassy

Volume 3 - Hadron Spin-Flip at RHIC Energies - BNL-64724 July 21 - August 22, 1997 - Organizers: T.L. Trueman and Elliot Leader

Volume 2 - Perturbative QCD as a Probe of Hadron Structure - BNL-64723 July 14-25, 1997 - Organizers: Robert Jaffe and George Sterman

Volume 1 - Open Standards for Cascade Models for RHIC - BNL-64722 June 23-27, 1997 - Organizer - Miklos Gyulassy 


\section{Preface to the Series}

The RIKEN BNL Research Center was established this April at Brookhaven National Laboratory. It is funded by the "Rikagaku Kenkysho" (Institute of Physical and Chemical Research) of Japan. The Center is dedicated to the study of strong interactions, including hard QCD/spin physics, lattice QCD and RHIC physics through nurturing of a new generation of young physicists.

For the first year, the Center will have only a Theory Group, with an Experimental Group to be structured later. The Theory Group will consist of about 12-15 Postdocs and Fellows, and plans to have an active Visiting Scientist program. A 0.6 teraflop parallel processor will be completed at the Center by the end of this year. In addition, the Center organizes workshops centered on specific problems in strong interactions.

Each workshop speaker is encouraged to select a few of the most important transparencies from his or her presentation, accompanied by a page of explanation. This material is collected at the end of the workshop by the organizer to form a proceedings, which can therefore be available within a short time.

T.D. Lee

July 4, 1997 


\section{CONTENTS}

Preface to the Series

Introduction

N. Saito and A. Schäfer . . . . . . . . . . . . . . . . . 1

RHIC Spin Physics

A. Schäfer . . . . . . . . . . . . . . . . . . 3

Event Generator for RHIC Spin Physics

N. Saito . . . . . . . . . . . . . . . . .

SPHINX - A Polarized Event Generator: Physical Foundations and Fu-

ture Developments of Event Generators

O. Martin

Event Generators

F. Paige .

Large $p_{T}$ Promt Photon and Photon plus Jet Production in Polarized $p p$ Collisions

L. Gordon

Event Generators for Special Processes: Examples of ep Collisions

M. Maul

Spin Physics with STAR

L. Bland

PHENIX Spin Physics

N. Hayashi

Polarized Parton Distributions and Event Generator

T. Shibata

An Introduction to Resummation and Intrinsic $p_{T}$

G. Sterman

Summary of the Discussion Session

N. Saito et al.

List of Participants

Workshop Agenda 


\section{INTRODUCTION}

This volume contains the report of the RIKEN BNL Research Center workshop on "Event Generator for RHIC Spin Physics" held on September 21-23, 1998 at Brookhaven National Laboratory. A major objective of the workshop was to establish a firm collaboration to develop suitable event generators for the spin physics program at RHIC.

With the completion of the Relativistic Heavy Ion Collider (RHIC) as a polarized collider a completely new domain of high-energy spin physics will be opened. The planned studies adress the spin structure of the nucleon, tests of the standard model, and transverse spin effects in initial and final states. RHIC offers the unique opportunity to pursue these studies because of its high and variable energy, $50 \leq \sqrt{s} \leq 500 \mathrm{GeV}$, high polarization, $70 \%$, and high luminosity, $2 \times 10^{32} \mathrm{~cm}^{-2} \mathrm{sec}^{-1}$ or more at $500 \mathrm{GeV}$. To maximize the output from the spin program at RHIC, the understanding of both experimental and theoretical systematic errors is crucial. It will require full-fledged event generators, to simulate the processes of interes in great detail.

The history of event generators shows that their development and improvement are ongoing processes taking place in parallel to the physics analysis by various experimental groups. The number of processes included in the generators has been increasing and the precision of their predictions has been being improved continuously. Our workshop aims at getting this process well under way for the spin physics program at RHIC, based on the first development in this direction, SPHINX.

The scope of the work includes:

- update of the currently existing event generator by including the most recent parton parametrizations as a library and reflecting recent progress made for spin-independent generators, - implementation of new processes, especially parity violating effects in high energy $p p$ collisions, - test of the currently available event generator by comparing to existing experimental data and analytical calculations for the unpolarized case, and

- search for ways to improve the treatment of polarization for the fragmentation phase.

In these directions, we have distributed several home works to the par- 
ticipants as shown in the summary of this workshop. The activities will be continued until and finalized in the upcoming month-long workshop to be held in next March. We expect that a month of concentrated work will provide a solid basement for rapid progress in this field such that reliable Monte-Carlo Codes will be available by the time the RHIC spin program starts to take data.

Naohito Saito and Andreas Schäfer are grateful to all participants of the workshop for their contributions on the various theoretical and experimental issues to be clarified by our collaboration. We would like to express our sincere thanks to the secretary of RIKEN BNL Research Center, Pam Esposito, for her great help in organizing and running the workshop. We want to extend our gratitude to Brookhaven National Laboratory and to the U.S. Department of Energy for providing the facilities to hold this workshop.

Naohito Saito (RIKEN / RIKEN BNL Research Center) Andreas Schäfer (Universität Regensburg)

RIKEN BNL Research Center

October, 1998 


\title{
RHIC SPIN PHYSICS
}

\author{
Andreas Schäfer
}

(Universität Regensburg)

\begin{abstract}
The situation of hadron spin physics is reviewed with respect to the special opportunities opened up by RHIC.
\end{abstract}




\section{Twist-2 Distribution Functions}

The three polarized lepton-nucleon scattering experiments SMC, HERMES, SLAC have determined $g_{1}^{p}\left(x, Q^{2}\right)$ rather precisely. The situation for $g_{1}^{n}\left(x, Q^{2}\right)$ is more involved (possible nuclear correlations in $\left.{ }^{6} \mathrm{Li} \uparrow\right)$. First semi-inclusive data become available. This allows to determine $\Delta u_{V}(x)$ and $\Delta d_{V}(x)$ quite well. The other distributions are hardly constraint $(\Delta \bar{u}, \Delta \bar{d}, \Delta \bar{s}$, $\Delta G$ ). The evolution of the angular momentum distributions is by now better understood. Although the Soffer bound for $\delta q$ survives $Q^{2}$-evolution, the predicted $\mathcal{A}_{T T}$ asymmetry for RHIC is marginal. The small- $x$ behaviour is not understood such that the first-moments of the distribution functions are poorly known.

Polarized proton-proton collisions at RHIC, analysed with the help of improved event generators will substantially improve our understanding of the polarized twist-2 distribution functions. 


\section{Higher Twist and Single Spin Asymmetries}

Higher Twist correlators constitute the bridge between perturbative QCD and more traditional hadron and nuclear physics. It can be analysed in the framework of exact $\mathrm{QCD}$, as opposed to various unclear forms of phenomenological model building often encountered in traditional nuclear physics. Substantial progress was made in the last years to relate single spin asymmetries to specific QCD correlators and to calculate their values in Lattice QCD. Also successful semi-empiric parametrisation of their form were developed. A better understanding of higher-twist correlators in general could be crucial to interpret the RHIC heavy ion reactions correctly.

RHIC is probably the best place to learn more about higher QCD correlators in the nucleon. 
RHIC Spin Physics

Andres Setiáfer (Regens bung)

What is the situation? what can Rule do?

What can we de?

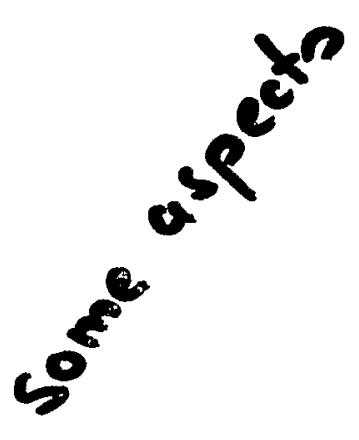

- status of the polarized twist-2 distribution functions

$\Delta u, \Delta \bar{u}, \Delta d, \Delta \bar{d}, \Delta s, \Delta \bar{s}, \Delta G$

$$
L_{q}(x), L_{G}(x), h_{1}(x)
$$

- Higher Twist Correlators

- Single Spin Asymmetries

6 
Iwist - 2

- In pol. Leptan-hadeon seatlering $g_{1}^{P}\left(x, a^{2}\right)$ is measuwed with vewy high preceission $g_{1}^{n}\left(x, Q^{2}\right)$ is mecesure! with quece! perecission First results for semi-inclusive recetions

- Nlo analysis $\rightarrow$ sul ade gaite good $\Delta \bar{u}, \Delta \bar{d}, \Delta s, \Delta \bar{s}, \Delta C$ hedly biencewn

- Small-x bebacieune of $\boldsymbol{H}_{1}$ is uncteavo $\rightarrow$ precise test of Bjorken - Sum Rule is very difficen lit.

- $\Delta \bar{u}-\Delta \bar{d}$ could be sizeable

- New evolution studies

$$
\left.\Rightarrow L_{G}(x) / A G(x)\right]^{7} \text { large } 0.1 \sim x=0.1
$$


- Soffer bound for $\delta g_{f}$ survives Q ev olution

- ATt fow RU!C disconergingly smo!! direet $\gamma^{\prime s}, w^{ \pm \prime}, Z$, jets, $n^{+} n^{-}$-( earge $\left.p_{1}\right)$, Dnell-Yan, ... $\Rightarrow \begin{aligned} & R S C \text { is needed to settle ! } \\ & \Delta q_{f}, \Delta \bar{q}_{f} ; \Delta G,\left(L_{q, G}(x) ?\right)\end{aligned}$

Requirement:

Comparisen of

NL.O parten gronecator with PYTM!n like. jensecutors has to be expanded jets SPHINX prompt $\gamma$ 's has to be -.

8 improved! 
Higher Iwist

- higher twist cerrelotors are of fundemental importance

$\Rightarrow$ systemetic stops towerel a honest QCD wevefunetion of the nueleen

- Two sebeels of thought

1.) transueve mementure effects derainate

$$
f\left(x_{0}, k_{1}, c^{2}\right)
$$

2.) Field cavelaters deminate

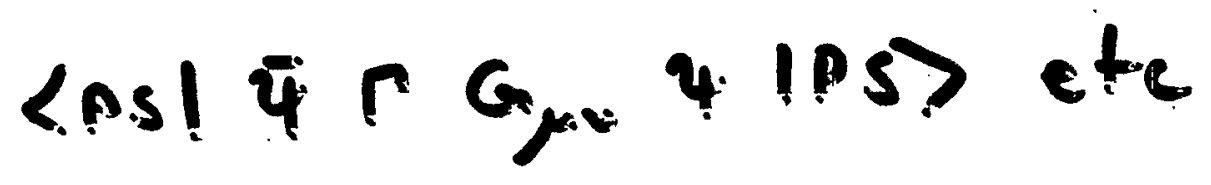

$\Rightarrow$ Highly relevant for RHIC heavy ion physics

9 
lollice calculations make pro guess to solve the problem of operator mixing

$\Rightarrow$ towards a lattice determination of the vel event coreletors

- $d_{2}$ : new lather calculations ave nefeleel

- Many single - spin- asymmetries are by new better understonel, Bog. a now rove SSA for Dull Yam in

$$
p+p^{\text {patrons! }} \rightarrow \mu^{+}+x
$$

con exist.

$p^{t}+p^{(t)}$ at Rule opens up unique possibilities

Reliable event generators are

10 needed to make use of this opportunity 


\section{RIKEN BNL Research Center Workshop on EVENT GENERATOR FOR RHIC SPIN PHYSICS}

September 21-23, 1998, BNL

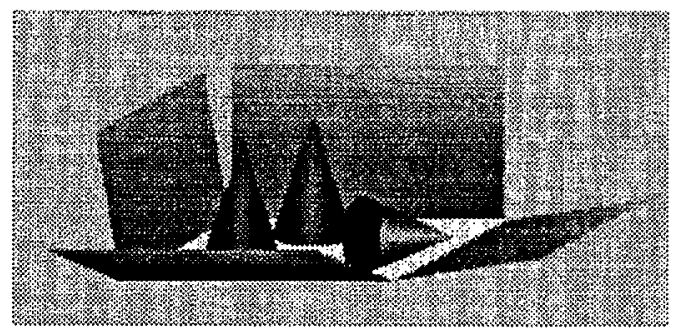

Naohito SAITO

RIKEN / RIKEN BNL Research Center

Naohito Saito, RIKEN BNL

\section{Needs of Event Generator}

from experimentalist's viewpoint

- Experimentalists can touch only Initial and Final state particles

- parton emission $f_{a / A}(x)$

- initial state radiation

- hard scattering (may include new physics) $d \sigma / d t$

- final state radiation

- fragmentation $D_{C / d}(z)$

- decay

- underlying event

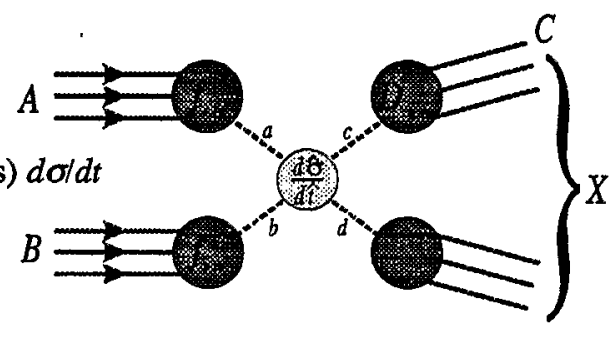

- Experimentalists often $\operatorname{aim} f_{a / A}(x), d \sigma / d t, D_{C / c}(z)$ to find new physics

- sensitivity for new physics / background studies

- geometrical acceptance for FSPs from physics of interests

- systematic uncertainties in interpretation of results

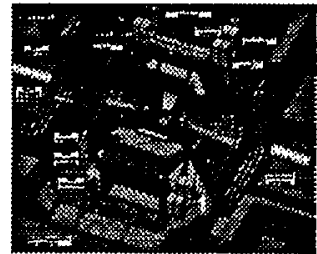




\section{Spin Physics at RHIC}

- Spin Structure of the Nucleon

$-\Delta g(x)$ : Gluon polarization via $\gamma, \pi^{0}$, heavy quark productions

$-\Delta \bar{q}(x):$ Anti-quark polarization via Drell-Yan $\left(W, Z, \gamma^{*}\right)$

$-\Delta_{T} q(x), \Delta_{T} \bar{q}(x)$ : Quark transversity

- Symmetry Tests

- parity violating effects, e.g. compositeness

- QCD Selection Rule

- switch off gluon; $a_{T T} a_{L L}<<1$

- Single Transverse Spin Asymmetry $A_{N}$

- large at low Energy; higher-twist? ; $k_{T}$ asymmetry?

Naohito Saito, RIKEN BNL

\section{Goals of this Workshop}

- Define scope of works until next March

- we will meet again for a month-long workshop

- Goals proposed - need your inputs

- reflect PYTHIA (v6.115) improvements to SPHINX

- implement new pol-PDF

- implement new process, parity violating process

- test SPHINX/PYTHIA against exp. data and NLO calculations

- search for a good way to handle polarization in the fragmentation phase

- $A_{N}$

- Intrinsic transverse momentum $p_{T}$ 


\section{Intrinsic $p_{T}$ : origin and effects on $g(x)$ $\rightarrow$ George Sterman's Talk}

- Both $k_{T}$ and $g(x)$ will affect cross section

- $k_{T}$ of order $1.0 \mathrm{GeV}$ required to reproduce fixed tgt exp Data

- What is the origin of $k_{T}$ ?

- Soft gluon emission

- Any energy dependence?

-Yes, $\ln (s)$

-What is the reasonable size of $k_{T}$ at RHIC?

- Could be as large as $3 \mathrm{GeV} / \mathrm{c}$ if $\ln (s)$.

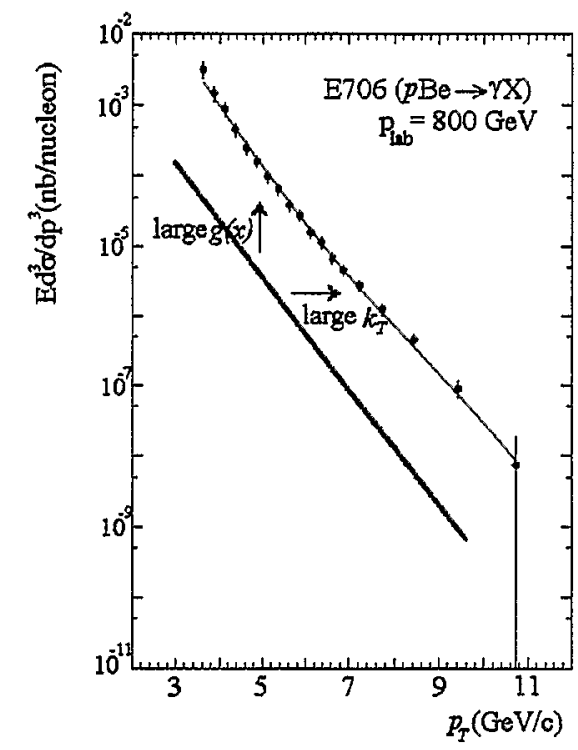

Naohito Saito, RIKEN BNL

\section{Unpolarized Gluon Distribution}

- Comparison of the most recent analysis normalized to MRST central

- MRST distribution

- hep-ph/9803445

- 3 versions of $g(x)$ $\left\langle k_{T}\right\rangle=0.0,0.4,0.64 \mathrm{GeV}$

- MRST $(\mathrm{g} \uparrow)$ high gluo

- MRST central

- MRST(g $\downarrow$ ) low gluor

- CTEQ4M

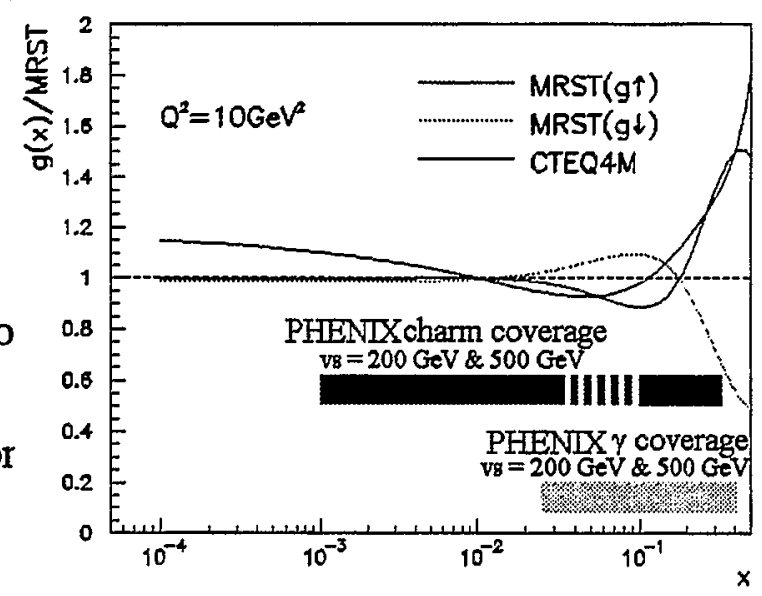

- PR D55 (97) 1280

Naohito Saito, RIKEN BNL 


\section{Parity Violation Beyond SM}

P. Taxil, J.M. Virey PLB 364(1995)181-187

PV in Jet Production due to quark Compositeness (scale: $\Lambda$ )

$\rightarrow$ should be reflected to single particle production
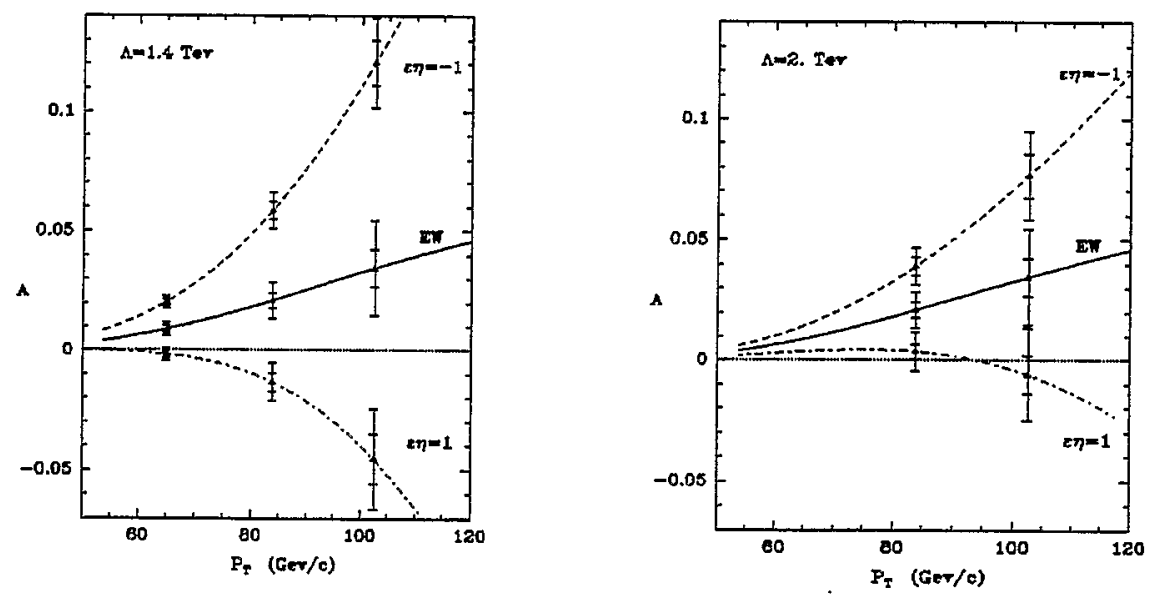

Naohito Saito, RIKEN BNL

\section{Charmonium Production}

Asymmetry Prediction with Color Octet Model

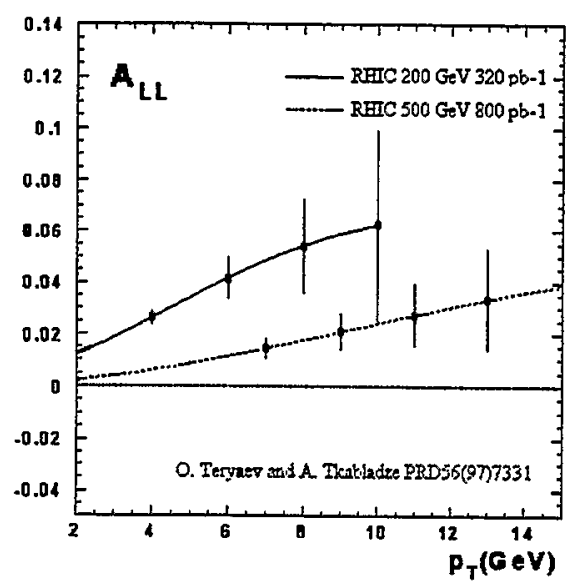

Yield Estimation with Color

Octet Model by Naoki

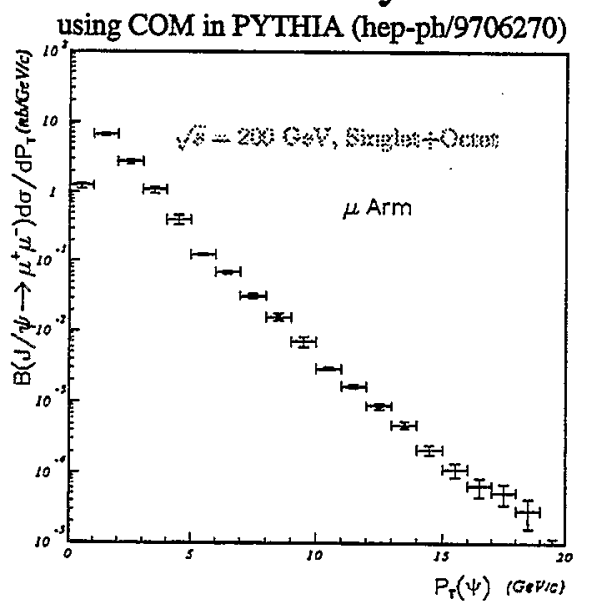

See D. Kharzeev's transparencies at Spin Discussion at http:/rikweb.rhic.bnl.gov/rsc/write-up/Kharzeev/kharzeev1.html Naohito Saito, RIKEN BNL 


\section{Single Transverse Spin Asymmetry}

\section{$A_{N^{-}}$higher twist effect}

- FNAL-E704 $p p$ collision at $\sqrt{s} \approx 20 \mathrm{GeV}$
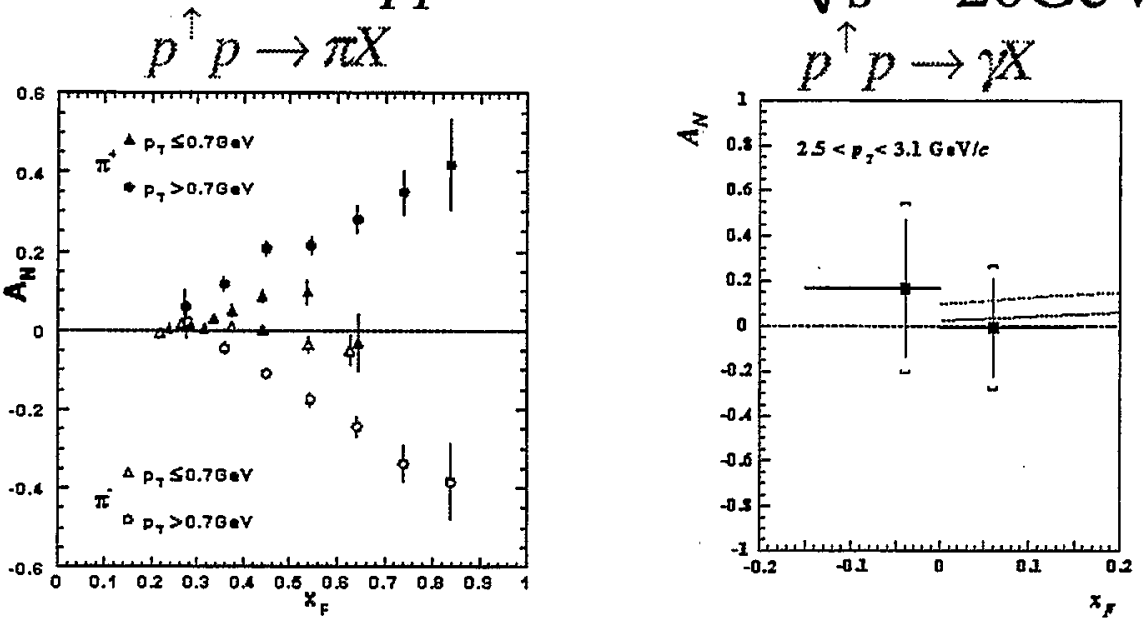

Naohito Saito, RIKEN BNL

\section{Spin Dependent Fragmentation to measure $\Delta_{T} q(x)$}

\section{R.L Jaffe, Xuemin Jin, Jian Tang hep-ph/9807560}

- Asymmetry $A_{\perp} \propto \Delta_{T} q \otimes a_{T T}^{i f} \otimes \Delta_{T} \hat{q}$
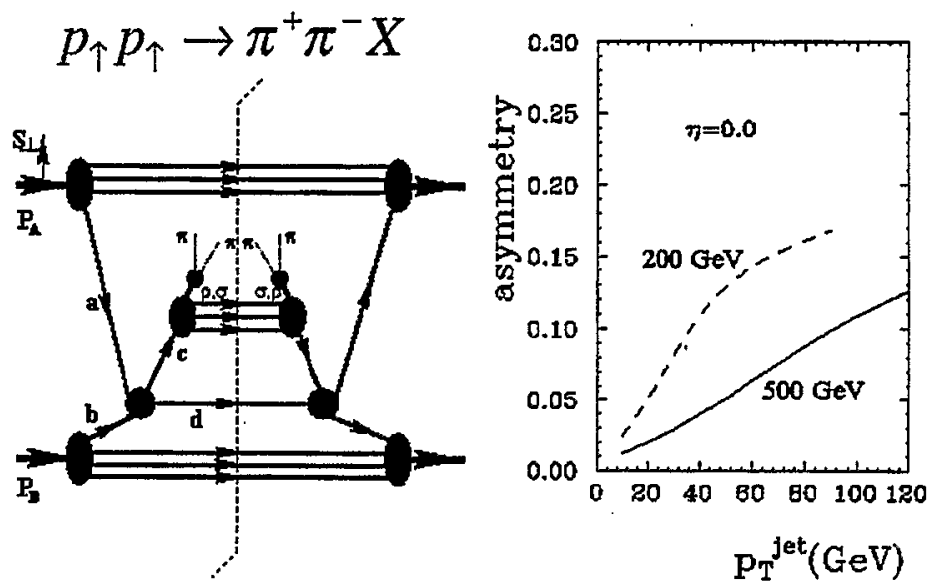

Soffer's inequality assumed to be saturated

Naohito Saito, RIKEN BNL 
.SPHINX - A Polarized Event Generator

Physical foundations and future developments of event generators

Oliver Martin and Andreas Schäfer,
Institut für Theoretische Physik, Universität Regensburg, Germany

The notion Monte Carlo (MC) actually describes two different types of programs with different philosophies. The first one should better be called parton-level MC and is aimed at calculating well defined LO or NLO QCD cross section with arbitrary phase space cuts. Here, the MC technique is only used for integration. Parton-level MCs have the advantage that they are based on a systematic perturbative expansion and contain very few free parameters. Because of the problem of negative weights they cannot be used as a simulation which generates events with the same probability as in nature. Such programs are called MC event generators. They are able to describe the full hadronic final state using LO pQCD and nonperturbative methods (hadronisation).

Event generators are based on the fact that in the collinear limit the NLO real corrections to the partonic cross sections can be written as product of the LO cross section times a splitting function. Based on this observation, they first generate the partonic interaction based on the LO cross-section formula and generate parton cascades for each external coloured particle. The cascades yield partons with a low virtuality which are then fragmented into hadrons. Unfortunately the parton cascades contain several free parameters and the meaning of some of the involved variables is not clear (What is the scale of the shower? What is the exact meaning of the splitting variable $z$ ? How large is the maximum virtuality and the infra-red cut-off? What is the argument of the strong coupling?). Due to colour coherence the opening angles in the parton cascades are strongly ordered, with the largest opening angles right at the hard partonic interactions. Each of these points can have an important impact on the numerical results.

Our codes Sphinx and Sphinx TT are based on the event generator Pythia 5.6 and allow the simulation of hadronic collisions of longitudinally and transversely polarized hadrons. Polarization is treated correctly in the initial state parton shower (ISS) and a wide variety of partonic matrix elements, whereas the final state shower and the fragmentation are done as usual. Since Pythia 5.6 is outdated by now the codes should be upgraded to Pythia 6.1 during this workshop. At the same time an increase in program speed would be welcome. Often signals as small as $1 \%$ have to be calculated which requires the generation of several million events taking days of computing time. However we found that we seem to get the same results if we do the. ISS without polarization so that we could simply generate unpolarized events and calculate the asymmetry afterwards. This method has several advantages, e.g., we can use several polarized parton distributions simultaneously, leading to a huge increase in speed. Furthermore the code would be independent of the actual version of Pythia allowing for an easy upgrade procedure.

One of the major problems of event generators is that they are only based on LO QCD matrix elements and that the parton showers underestimate hard emission. Furthermore some angular distributions are not well described. These problems can be cured by also taking into account NLO QCD real correction matrix elements with well seperated partons and using them as input for the final state shower generator (forced parton showers). An implementation of forced parton showers in Sphinx would be a significant improvement. 
Sphinx / Sphinx TT

Features

- based on Pythia 5.6 / Jetset 7.3 ( 1992)

- Longitudinal / transverse polarization included in

- pdf

- initial state shower (no nonlocal angular correlations)

- ME (incoming partons)

- Available partonic subprocesses

\begin{tabular}{lcc} 
& Sphinx & Sphinx TT \\
\hline high PT QCD & $\checkmark$ & $\checkmark$ \\
direct photons & $\checkmark$ & $\checkmark$ \\
Drell-Yan & $\checkmark$ & $\checkmark$ \\
$Z^{\circ}, \omega^{+/}$production & $\checkmark$ & \\
photoproduction & $\checkmark$ &
\end{tabular}

- Implementation:

Just split flavor $a$ into $\left(a_{1}+\right),\left(a_{1}-\right)$ and treat them as different flavors. Substitute pdf, splitting functions and ME by polarized counterparts.

17 
Problems / Wishlist

- Speed! Asymmetries of $\partial(1 \%)$ are common. We need $10^{6}$ events / bin to achieve 10\% accuracy (IF each event fulfils cuts!)

PPr 200 based computer generates approx 400000 events per hour.

Improvable? Maybe.

- Only leading Twist. (Twist-2)

$\Rightarrow$ only double spin asymmetries, no $A_{L T}$

- No polarization in final state shower and hadronisation. Important? Implementation of polarized FSS easy. No hadronisation model available which includes spin.

- based on old Pythia/zetset version from 1992 $\Rightarrow$ upgrade to Pythia 6.1. Lots of work...

- Move?

18 
- How can we improve the speed?

Do results notably depend on polarization in ISS?

$$
\text { No!? }
$$

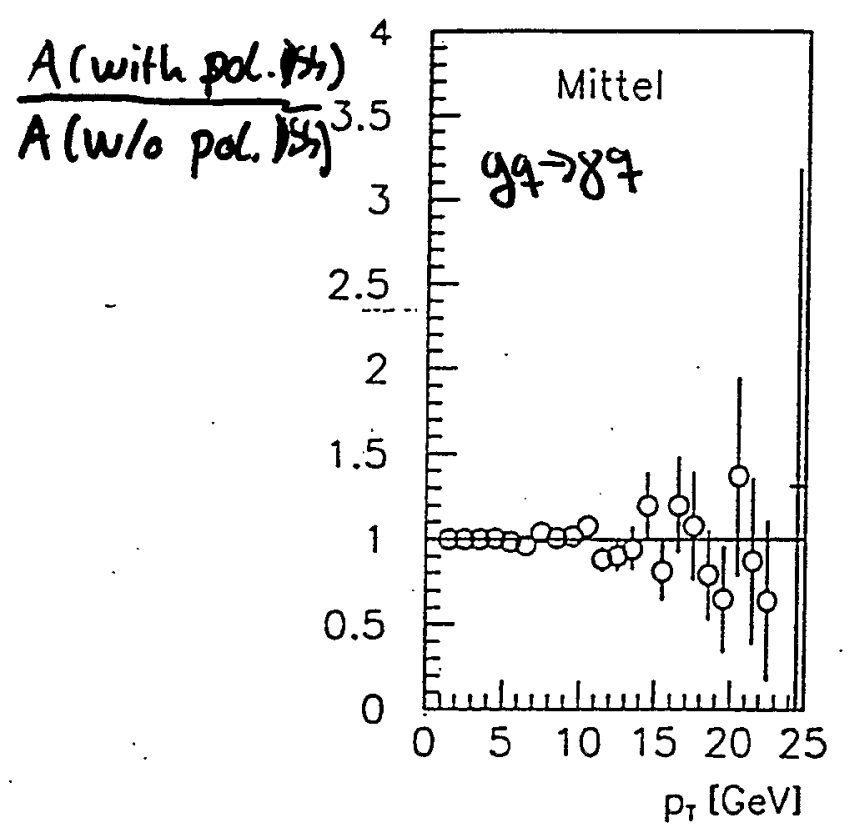

New procedure:

- calculate un polarized event (determine $x_{a}, x_{b}, \hat{s}, \hat{t}, \hat{u}$ )

- calculate $A=\frac{\Delta f_{a}\left(x_{a}\right)}{f_{a}\left(x_{a}\right)} \frac{\Delta f_{b}\left(x_{b}\right)}{f_{b}\left(x_{B}\right)} \frac{\Delta \hat{\sigma}(\hat{s}, \hat{t}, \hat{u})}{\hat{\sigma}(\hat{s}, \hat{t}, \hat{u})}$

- after all events are generated: $\Delta \sigma=A \cdot \sigma$ (histo. level)

Advantages:

- much faster convergence

- several sets of polarized poof can be used simultaneously

- fast implementation

- little support necessary, since Pythia version can be easily upgraded

- maybe also Herwif can be used as event generator

19 
- First test of new method

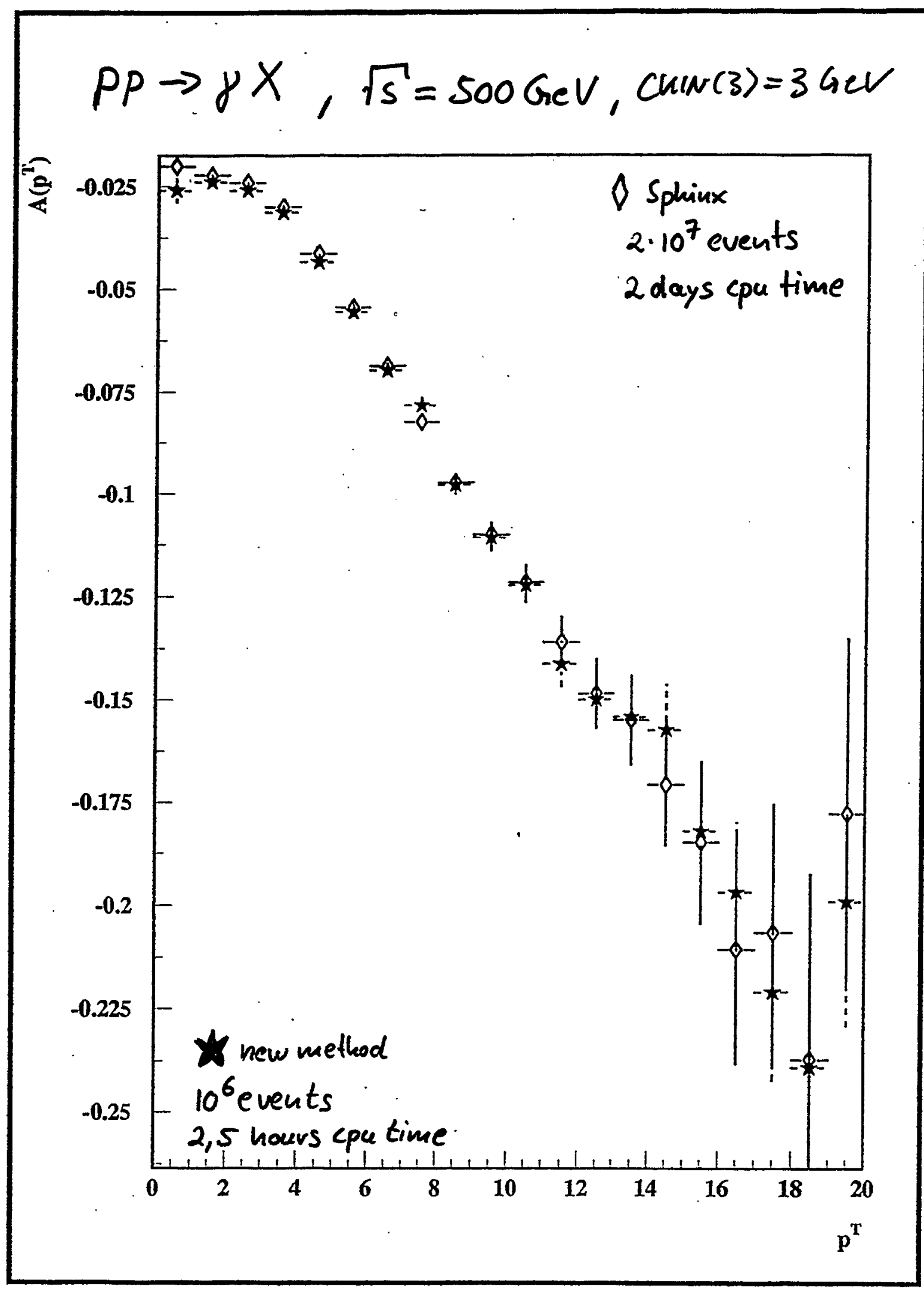

20 


\section{Event Generators \\ Frank E. Paige \\ Brookhaven National Laboratory \\ Upton, NY 11973}

Main goal of RHIC spin physics is to measure parton spin distributions $\Leftrightarrow$ measure hard processes. Need to relate data to partons. Large effects, since detectors not optimal. But only need asymmetries. Main tool is parton shower Monte Carlo event generators (e.g., HERWIG, ISAJET, PYTHIA).

Main limitations of current generators:

- Based (mainly) on leading-log QCD. Extension to NLO highly non-trivial. C.f. Sjostrand.

- Poor understanding of beam jets. C.f. Webber

Straightforward to add polarized parton distributions and cross sections. Cannot just use generator to extract NLO spin distributions. Unlikely to improve soon.

Structure of jets mainly controlled by pQCD. Soft hadronization effects small. But hard scattering uses small fraction of $\sqrt{s}$. Rest goes into low- $p_{T}$ particles. No good theory for this or for minimum bias events.

DTUJET Monte Carlo for minimum bias well tuned to data and not obviously wrong. Basis idea is to use eikonal to combine semihard jets and soft physics:

$$
f(s, t)=2 i s \int d^{2} b e^{i k \cdot b}\left[1-e^{-\Omega_{h}(s, b)-\Omega_{s}(s, b)}\right]
$$

Expand and use AKG cutting rules.

- $\Omega_{h} \Rightarrow$ low- $p_{T}$ jet event, generated assuming standard gluon distribution.

- $\Omega_{s} \Rightarrow$ soft physics only.

Eikonal guarantees consistency with basic unitarity.

Important to study both multiple parton interactions and underlying events at RHIC. 


\section{Beyond Leading Log?}

Some possible strategies:

- Modify parton shower.

- Attach showers to tree-level multi-partons.

- Attach showers to NLO partons.

Sjostrand: "No solution in sight!"

\section{Modified Shower:}

Example: $e^{+} e^{-} \rightarrow q \bar{q} g$ in PYTHIA. Weight first parton shower:

$$
d \mathcal{P}=\frac{\alpha_{s}}{2 \pi} \frac{d Q^{2}}{Q^{2}} P(z) d z S\left(Q_{\max }^{2}, Q^{2}\right)
$$

to match exact matrix element:

$$
\frac{1}{\sigma} \frac{d \sigma}{d x_{1} d x_{2}}=\frac{\alpha_{s}}{2 \pi} \frac{4}{3} \frac{x_{1}^{2}+x_{2}^{2}}{\left(1-x_{1}\right)\left(1-x_{2}\right)}
$$

where

$$
x_{1,2}=\frac{2 E_{1,2}}{E}, \quad x_{3}=1-x_{1}-x_{2}
$$




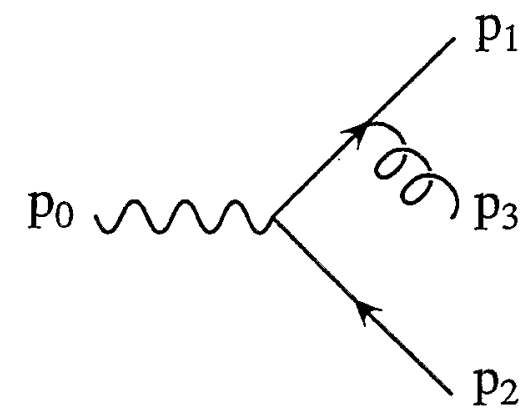

Radiation from 1 gives

$$
\begin{aligned}
Q^{2} & =\left(p_{0}-p_{2}\right)^{2}=\left(1-x_{2}\right) E^{2} \\
\frac{d Q^{2}}{Q^{2}} & =\frac{d x_{2}}{1-x_{2}} \\
z & =\frac{p_{0} \cdot p_{1}}{p_{0} \cdot\left(p_{1}+p_{3}\right)} \\
d z & =\frac{d x_{1}}{2-x_{2}}
\end{aligned}
$$

Symmetrize:

$$
\begin{gathered}
\frac{1}{\sigma} \frac{d \sigma_{\text {shower }}}{d x_{1} d x_{2}}=\frac{\alpha_{s}}{2 \pi} \frac{4}{3} \frac{A\left(x_{1}, x_{2}\right)}{\left(1-x_{1}\right)\left(1-x_{2}\right)} \\
A=1+\frac{1-x_{1}}{2-x_{1}-x_{2}}\left(\frac{x_{1}}{2-x_{2}}\right)^{2}+\frac{1-x_{2}}{2-x_{1}-x_{2}}\left(\frac{x_{2}}{2-x_{1}}\right)^{2}
\end{gathered}
$$

Generate with shower and reject with

$$
\frac{x_{1}^{2}+x_{2}^{2}}{A\left(x_{1}, x_{2}\right)} \leq 1
$$

Improves 3-jet distribution but still normalized to

LO cross section. 
D0 measured $\Delta \phi_{\mu \mu}$ distribution for $M_{\mu \mu}>6 \mathrm{GeV}$.

LO processes give $\delta$-function at $\Delta \phi_{b \bar{b}}=180^{\circ}$. NLO processes give both positive events with $\Delta \phi_{b \bar{b}}<180^{\circ}$ and negative $\delta$-function at $180^{\circ}$.

Can remove singularities and reduce weight fluctuations by combining events using resolutionsmeared $b$ and $\bar{b}$ :

$$
\chi^{2}=\sum\left(\frac{\Delta p_{T}^{2}}{\sigma_{p_{T}}}\right)^{2}+\sum\left(\frac{\Delta y^{2}}{\sigma_{y}}\right)^{2}+\left(\frac{\Delta \phi^{2}}{\sigma_{\phi}}\right)^{2}
$$

using reasonable $\sigma$ 's for experiment.

Matching is done in stages. First combine events and counterevents, averaging $p_{b}$ and $p_{\bar{b}}$. Then combine each negative event with first good match.

Finally combine each negative event with best match using looser cut.

Awkward procedure and requires $\mathcal{O}\left(N^{2}\right)$ steps.

Final negative event sample is $\sim 30 \%$ of total.

Feed resulting partons into parton shower, hadronization, and detector simulation. Final result: 


\section{D0 $\Delta \phi_{\mu \mu}$ distribution from Run Ia and NLO}

\section{HVQJET prediction:}
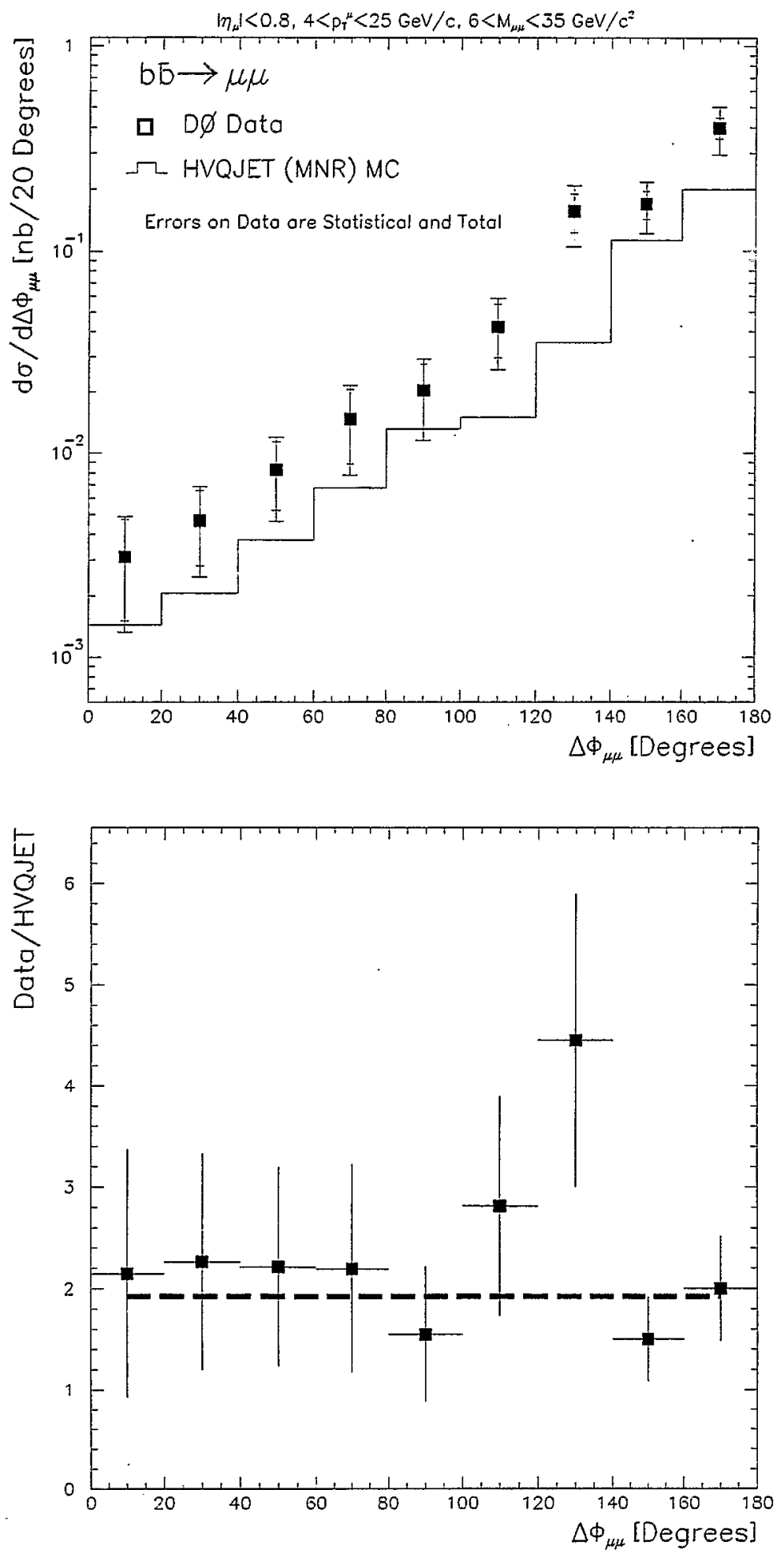


\section{CDF double parton scattering:}
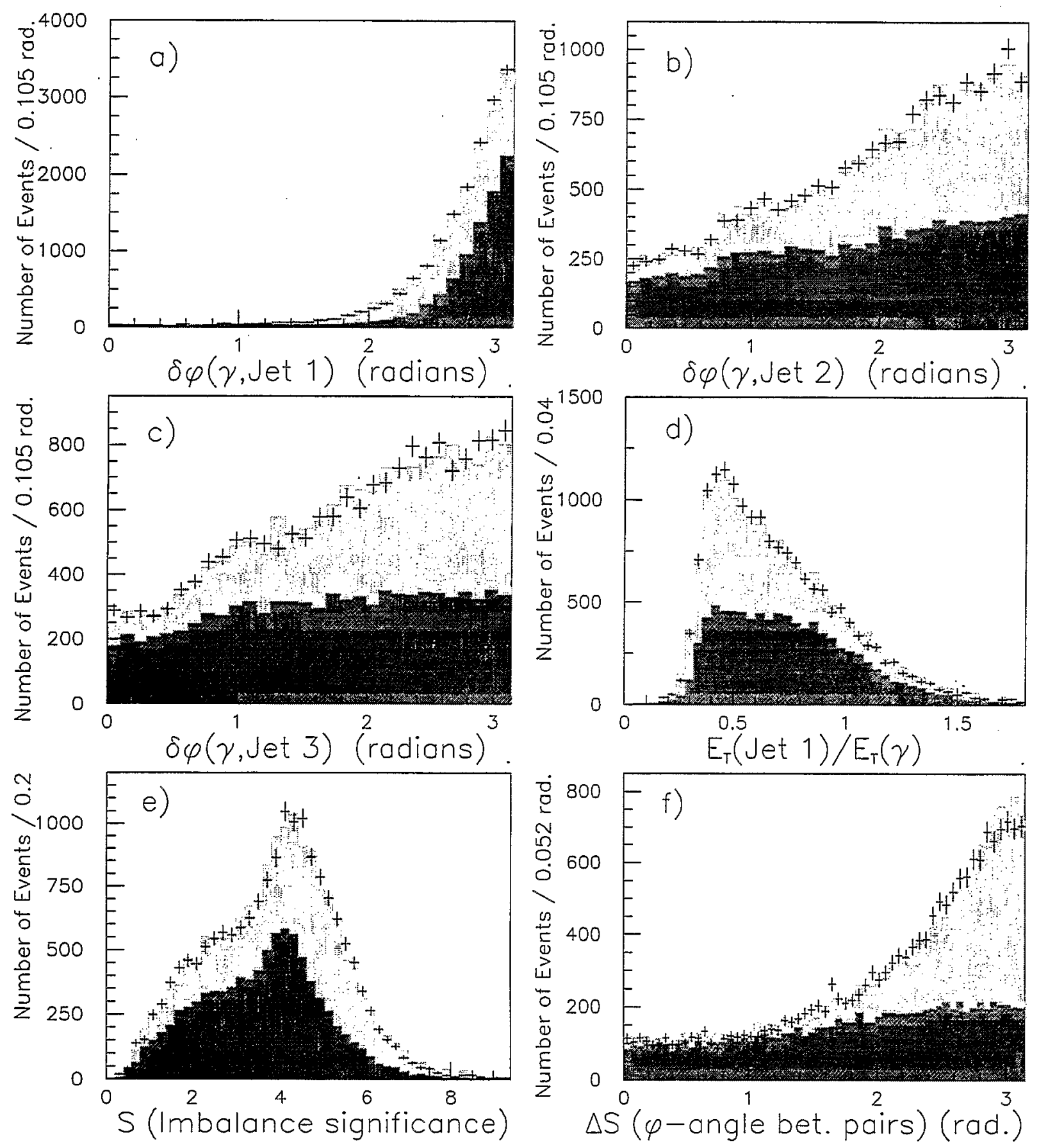


\title{
Large $p_{T}$ Prompt Photon and Photon plus Jet Production in Polarized $p p$ Collisions
}

\author{
L. E. Gordon \\ Jefferson Lab and Hampton University, Va
}

Polarized deep inelastic scattering experiments have yielded useful information on the spin structure of the nucleon, but the data are currently not precise enough to allow the spin dependent gluon distribution $\Delta G$ to be satisfactorily constrained. For this purpose other high energy scattering processes have been proposed for study in future experiments at RHIC and HERA. Prompt photon and photon plus jet production are currently two of the most promising processes proposed for extracting information on $\triangle G$ at both RHIC and HERA.

The processes $p p \rightarrow \gamma+X$ or $p p \rightarrow \gamma+$ Jet are dominated by the LO scattering subprocess $q g \rightarrow \gamma q$, and thus are very sensitive to $\Delta G$. Full next-to-leading order calculations for prompt photon and photon plus jet production have been performed using both analytic and Monte Carlo techniques. The dependence of the asymmetries on photon isolation, jet definitions and the choice of hard scales have been studied extensively. All such studies confirm the usefulness of the processes for extracting information on $\Delta G$. In general dependence of the asymmetries on uncertainties in these quantities is small.

In the unpolarized case the usefulness of prompt photon production for extracting the gluon distribution at collider energies has been restricted by uncertainties in the theoretical predictions in the low $x_{T}=2 p_{T} / \sqrt{s}$ region. This is the region where fragmentation contributions are most significant, even after isolation, and where soft gluon resummation may be necessary to improve the NLO theoretical predictions. It is expected the at RHIC energies similar problems will be encountered thereby reducing the usefulness of prompt photon production for extracting $\Delta G$ in the lower $x$ region. 


\section{Introduction}

Starting Point: What We Know About Nucleon Spin Structure from DIS

Current information from DIS:

Measurement of $g_{1}^{p}(x)$ at 'low' $Q^{2}$ yields info mostly on valence quark distributions

Still large uncertainties in $\Delta G$ and $\Delta s$ and even $\Delta \overline{(u, d)}$

E.g, new GGR Parametrization proposes 3 gluon (and sea) models

1. GGRA - moderate $\Delta G=x G$ at small $Q^{2}$

2. GGRB - zero $\Delta G=0$ at small $Q^{2}$

3. GGRC - instanton motivated: $\langle\Delta G\rangle<0$ at small $Q^{2}$ 



\section{Prompt $\gamma$ and $\gamma+$ jet Production}

These are two of the most promising processes to help determine $\Delta G$ at R.HIC and HERA- $\vec{N}$

Prompt $\gamma(+$ jet $)$ Production depends directly on $\Delta G$ in LO due to the dominance of the $q g \rightarrow \gamma q$ subprocesses

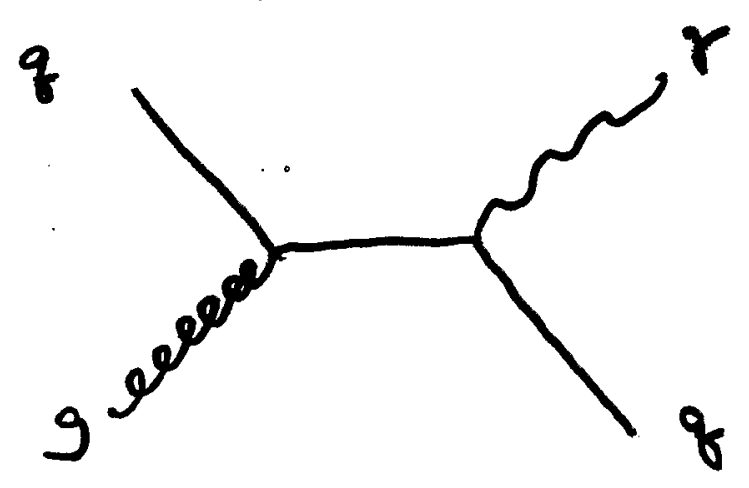

\section{Advantages:}

1. relatively large cross section

2. relatively clean signal (except at low $x_{T}$ )

3. NLO calculation done

A. P. Contogouris et al., '93 (Analytic)

L. G. and W. Vogelsang' 93 (Analytic + Isolation)

L. G. '96 (Monte Carlo) 
Leadizg Order QCD Calculation $O\left(\alpha_{s} \alpha_{e m}\right)$ at HadronFadron Colliders Involves:

(a) Non-fragmentation processes: $q g \rightarrow \gamma q$ and $q \bar{q} \rightarrow \gamma g$
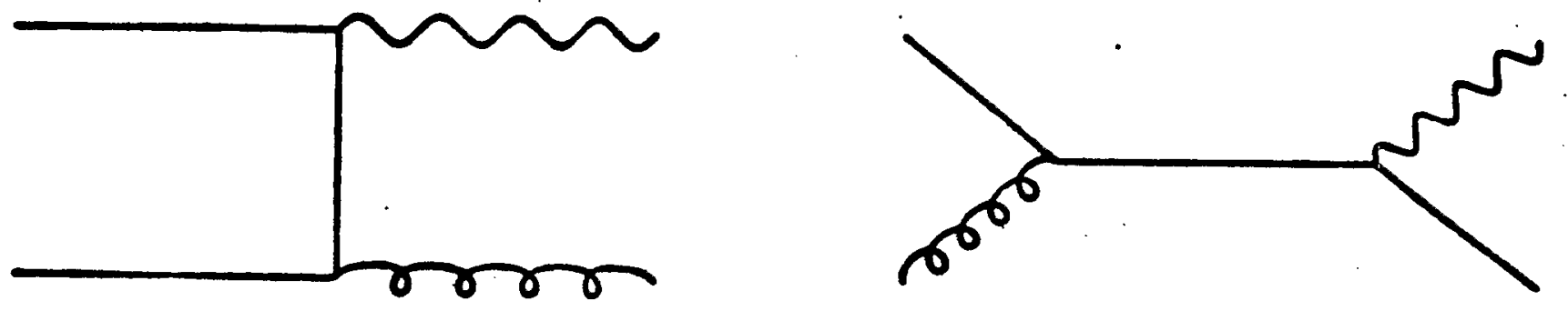

and (b) Photon Fragmentation Processes:

$$
\begin{aligned}
& q+g \rightarrow g+q \\
& g+g \rightarrow q+q \\
& q+q^{\prime} \rightarrow q \cdots q^{\prime}
\end{aligned}
$$

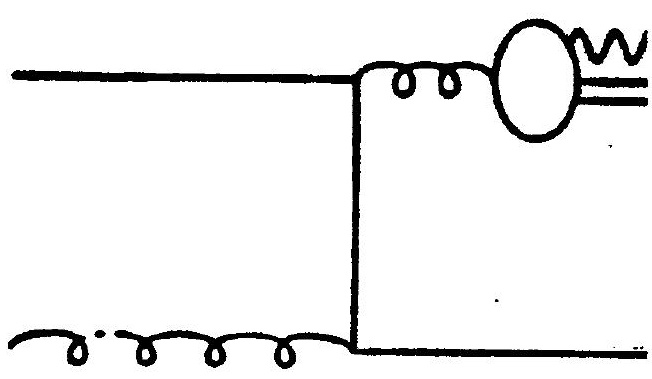

$D_{g / r}\left(+, \theta_{+}^{2}\right) \quad q+\bar{q} \rightarrow q+q$

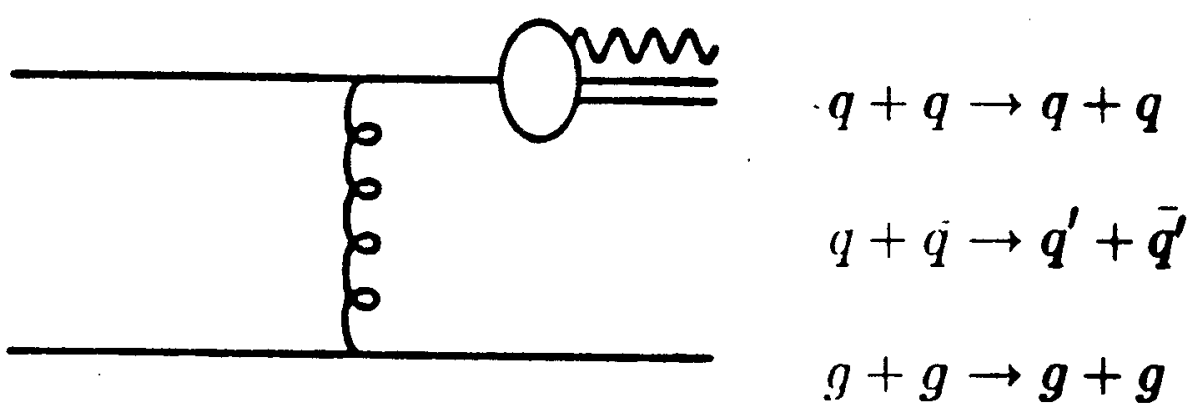




$$
A_{\text {LC }}=\frac{\Delta 6}{6}
$$

$$
p p \rightarrow \gamma+x, \sqrt{s}=
$$
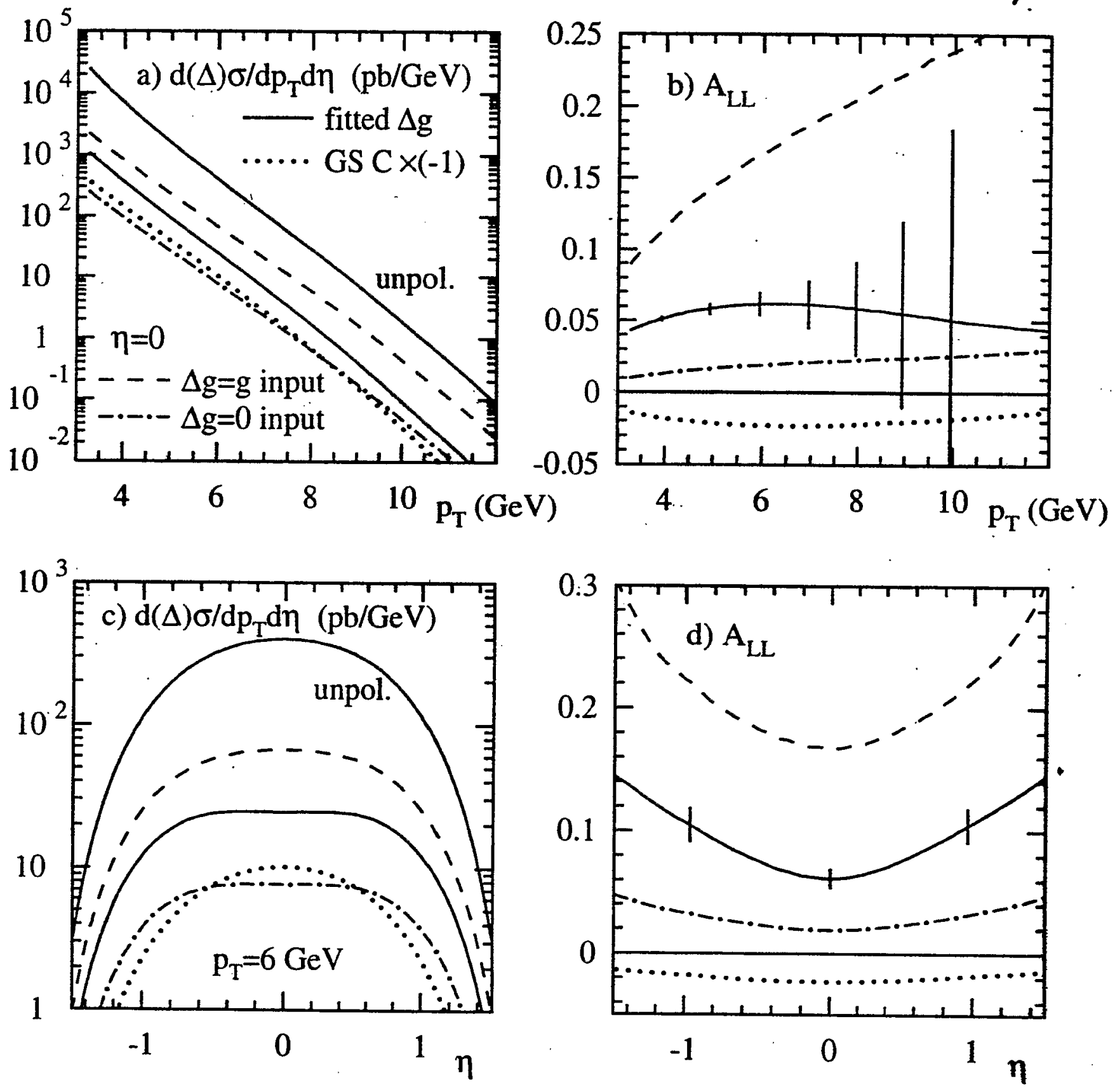

Fig. 2

$$
S A_{L C}=\frac{0.17}{\sqrt{6(P 6)}}
$$

$w \rightarrow$ Nowate

32 


\section{Conclusions}

(1) Large $p_{T}$ prompt $\gamma$ production and $\gamma+$ Jet production at RHIC and HERA- $\vec{N}$ will yield important information on nucleon spin distributions and structure particularly $\Delta G$

(2) Predictions show that asymmetry measurements in these processes will also allow distinction between various models of nucleon spin structure

(3) Measurement of all these processes are feasible at the sites of the planned polarization experiments.

(4) Problems with the theoretical predictions in the low $x_{T}=2 p_{T} / \sqrt{S}$ region will make the cross sections less useful at RHIC energies, but this will only affect the low $x$ region. Valuable information will still be obtained. 


\section{Summary for the Talk: \\ Event Generators for Special Processes: Examples for ep Collisions}

M. Maul, NORDITA, Blegdamsvej 17, 2100 Copenhagen, Denmark

In Addition to PHYTHIA/JETSET a variety of MC-generators have been developed which concentrate on special processes, but which make use also of the principles of string fragmentation and parton showering. The aim of these special generators is to concentrate on:

- NLO corrections

- higher twist corrections

- polarization of initial and final states

- radiative corrections.

For example, for polarized Deep Inelastic Scattering the MC generator PEPSI was developed to describe longitudinally polarized lepton nucleon collisions. It was used to investigate the possibility to measure the polarized gluon density, semi inclusive asymmetries and asymmetries involving charged current processes at HERA with polarized protons and electrons. PEPSI is a full MC-generator. NLO corrections are taken into account via interpolation tables to speed up the simulation velocity. Quark masses are taken into account via threshold effects only. Hadronization and parton showering are subsequently included by calling JETSET routines. On the parton level, neglecting quark masses we find good agreement with the integrator MEPJET.

For future pp studies, accompanying PYTHIA and SPHINX, the author suggests an integrator based event generator, interpolation tables for analytically feasible integrations should be used and subsequently hadronization models are to be added. As an example for such an event generator the program EPEPSI for the despription of polarized elastic meson pair leptoproduction is explained. The amplitudes over off-forward parton distributions are calculated analytically and stored in interpolation tables. The kinematic variables are chosen randomly and the cross section is calculated as a weight for the respective event. Partons are formed according to the kinematic configuration and subsequently the hadronization is done via JETSET. Cuts suitable for the corresponding detectors (in this case HERMES) can be applied on the generated event record.

Possible event generators for RHIC accompanying PYTHIA could be written for polarized Drell Yan, polarized semi inclusive pp collisions, single and double elastic pp collisions. 
2. The Principles of PEPSI

- Tree -cross section is monte corlio simulated exactly:

$x$ find the maximum

$x$ regent or accept kinematic configurations as to the maximum:

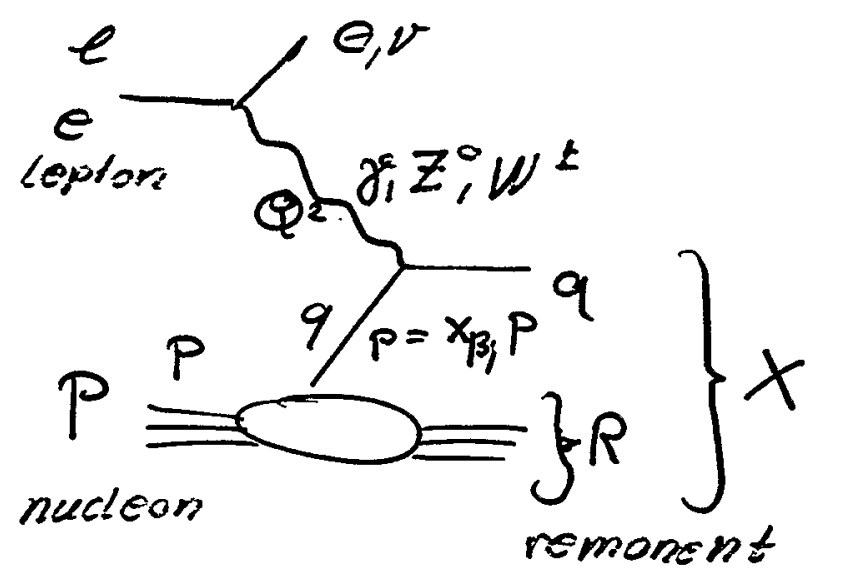

$$
\begin{aligned}
& y=\frac{P q}{P e} \\
& x_{B_{j}}=\frac{Q^{2}}{2 P q}
\end{aligned}
$$

only $y_{1} x_{B ;}$ ore independent variables

- NLO corrections are integrated before hand and are generated with respect to the tree cross section
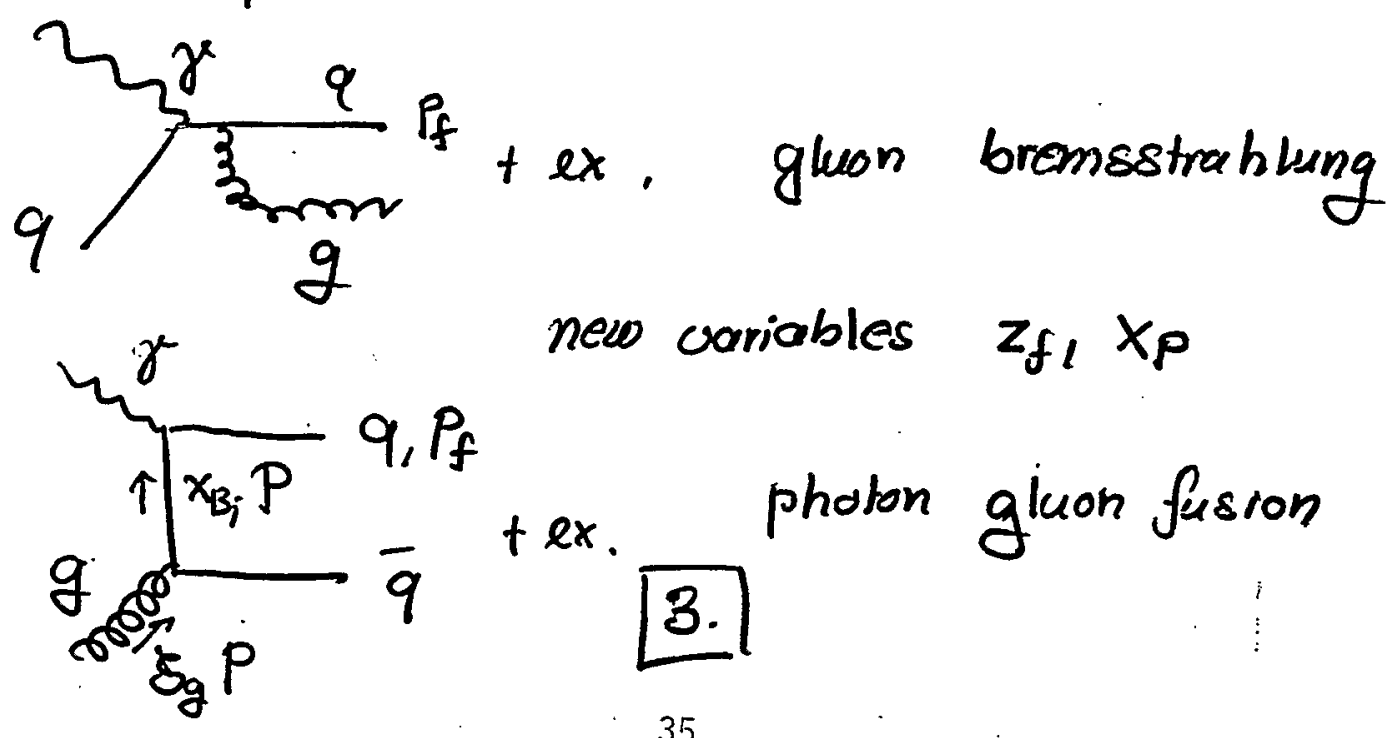

photon gluon fusion

3.

35 
- inclusion of higher durst effects by modifications of the portion distribution functions

- $\alpha_{s}^{2}, \alpha_{s}^{3}$ effects elk. by unpolorized portion showering

- fragmentation by JETSET

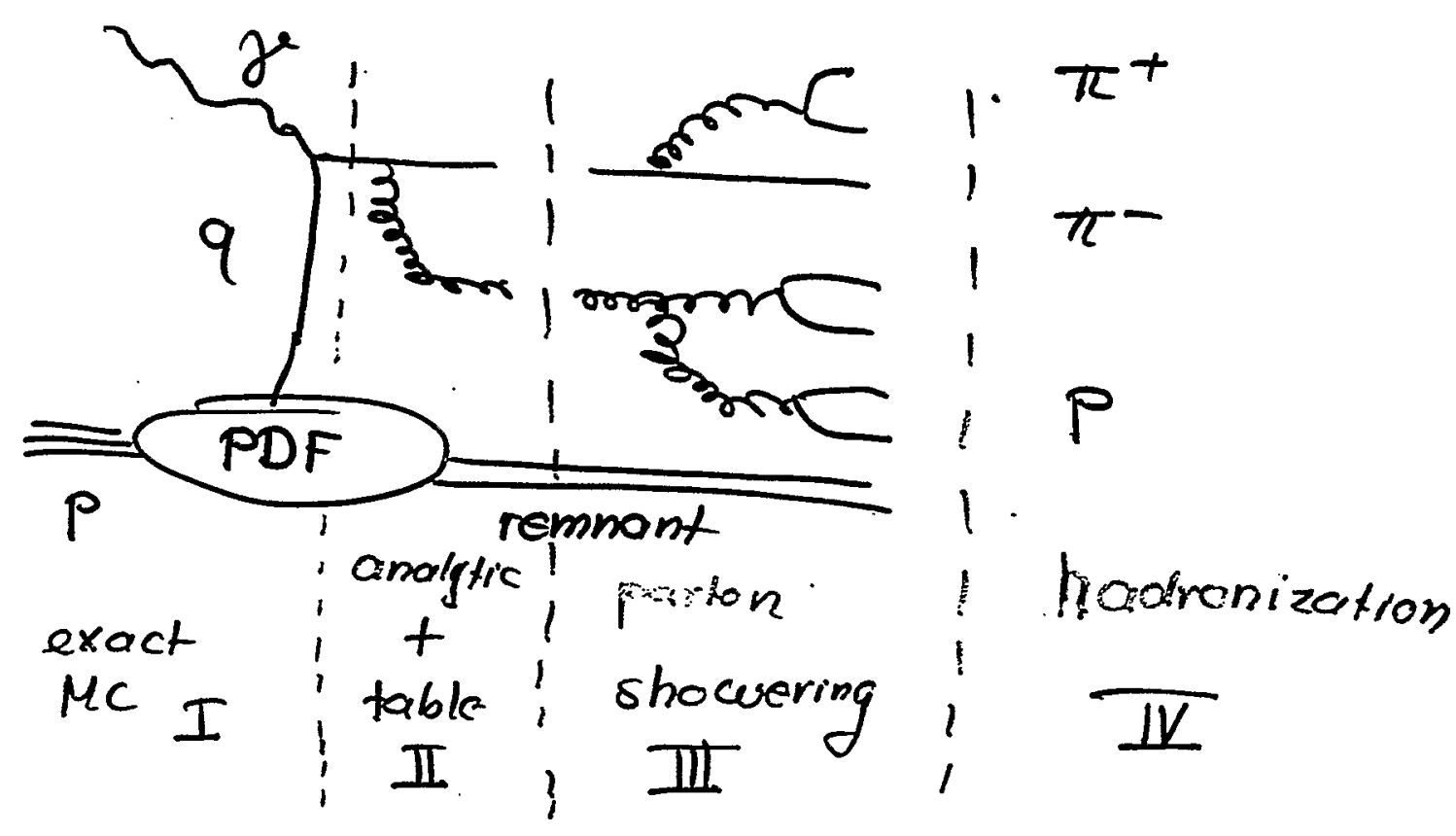

Problems: - The analytic integration in II requires a jet scheme which is in principle different from

5. the hodronic yet scheme in N !

- The $\alpha_{s}$ cross sections in II are moss less while the steps III and IV take quark masses into account.!

36 
3. The polarized MERA project:

Applications of PEPSI -2

A. Analyzing the polarized gluon density by dijet events

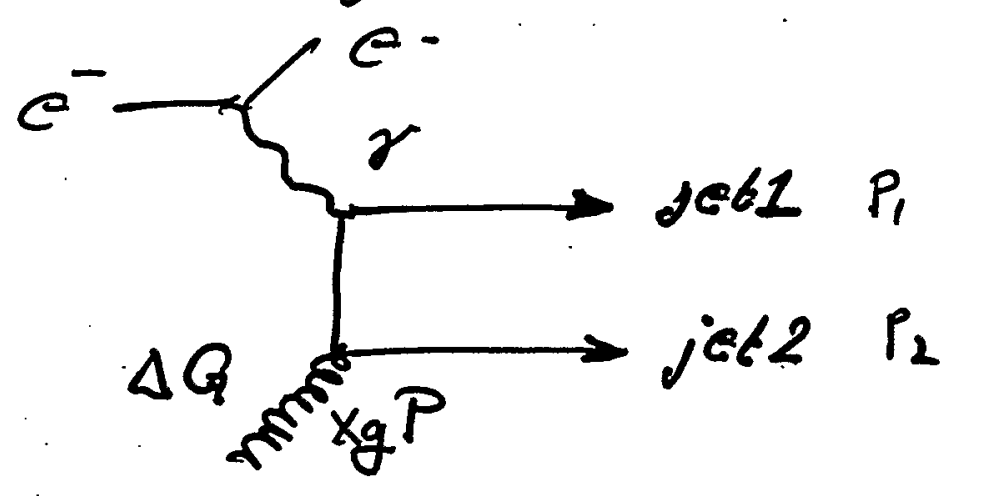

$$
s_{i j}=\left(p_{1}+p_{2}\right)^{2}
$$

- the photon gluon fusion is atchingating over the bremsstrahlung

- Comparison on the parton level with the MC -integrator MEPJET

MllEPJET : no quark masses (this reason)

PEPSI -2 : quark masses included only via threshold affects

$\Rightarrow$ exact agreement as to the asymmetries

6.

37 

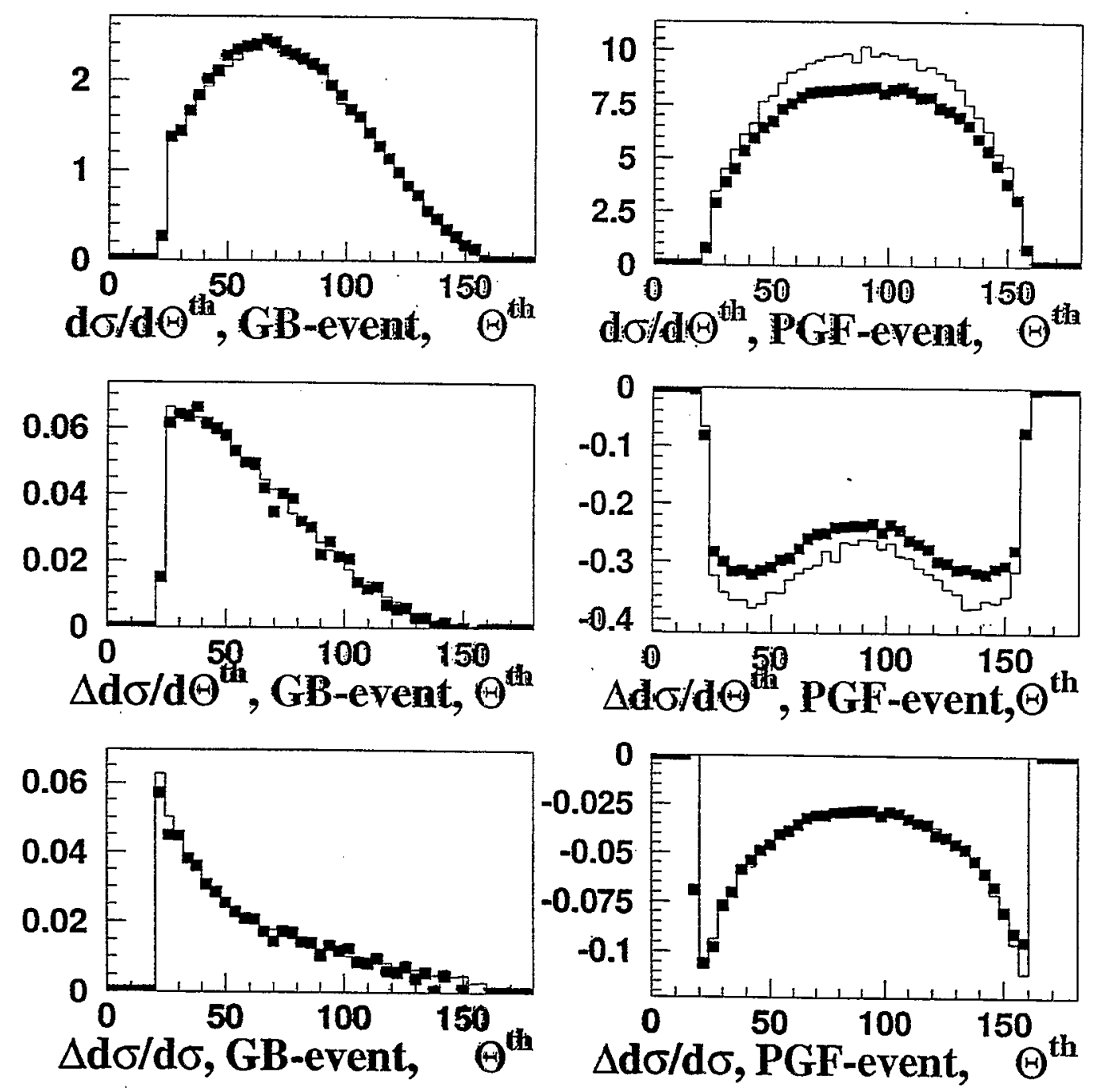

Abbildung 1: Influence of quark-mass effects to the unpolarized and polarized dijet crass sections. We show the results of the massless MEPJET program (histogram) and the PEPSI code (full squares), where quark-mass effects and remnantmass effects are included. The units are in $\mathrm{pb} /$ degree. 
Cir Simulations of semi inclusive

DIS with charged currents

Basic idea: missing momentum procedure

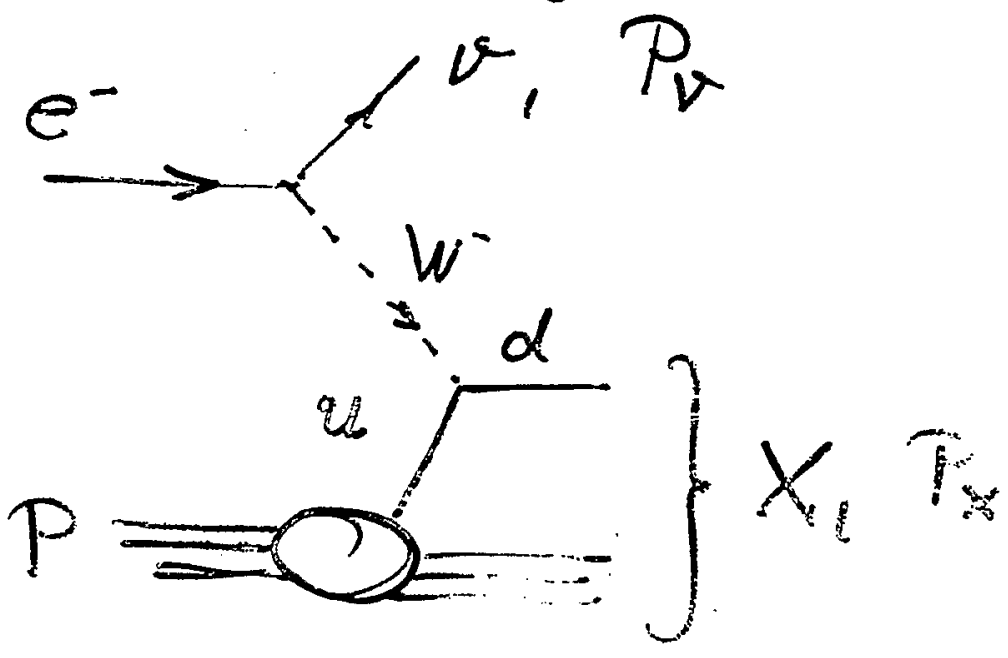

reconstruction of $P_{V}$ by a good measurement of $P_{X}$.

asymmetries: $\quad A\left(W_{,}^{-} h\right)=\left.\underbrace{\frac{1}{P_{e} P_{p}}}_{h_{i} w} \frac{\Delta \sigma^{h}}{\sigma_{n}}\right|_{W}$

$=\frac{\Delta u D_{u}^{h_{1} w}-(y-i)^{2}\left(\Delta \bar{s} D_{s}^{h_{s} w}+\Delta \bar{d} D_{\bar{d}}^{h_{1} w}\right)}{u D_{u}^{h_{1} w}+(y-1)^{2}\left(\bar{s} D_{s}^{h_{c} w}+\bar{d} D_{\bar{d}}^{h_{1} w}\right)}$

- $d\left(w_{1}^{-} \pi^{+}\right), d\left(w_{1}^{-} \pi^{-}\right)$differ only with respect to the antistrange quark contribution

9.

39 

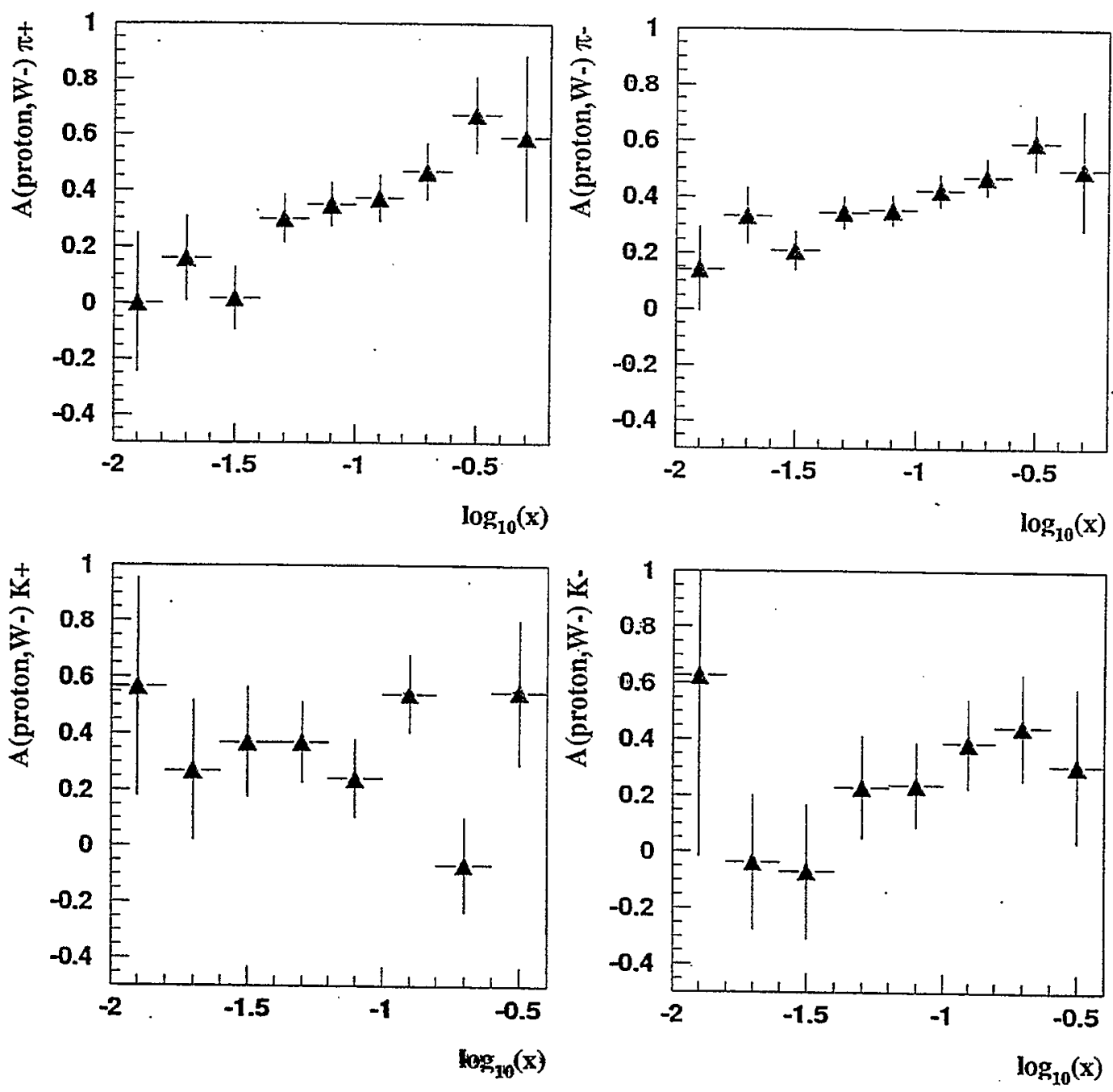

Abbildung 11: Semi-inclusive asymmetries $\frac{1}{P_{e} P_{p}} \frac{\Delta \sigma^{H}}{\sigma^{H}}, H=\pi^{+}, \pi^{-}, K^{+}, K^{-}$for $W^{-}$ exchange, for $200 \mathrm{pb}^{-1}$ per relative polarization and polarization degree $P_{e}=$ $P_{p}=$ 0.7. Parton distribution set GRSV LO STD. Simulation done with PEPSI. 


\section{Spin Physics with STAR \\ L.C. Bland \\ Indiana University Cyclotron Facility, Bloomington, IN USA}

Collisions between longitudinally polarized protons beams at RHIC in the energy range $100 \leq \sqrt{s} \leq 500 \mathrm{GeV}$ will provide a world-class facility for the study of the spin structure of the nucleon. Both the PHENIX and STAR detectors will be used for the studies of parity allowed two-spin asymmetries $\left(A_{I L}\right)$ and parity-violating single-spin asymmetries $\left(A_{L}\right)$. Of the two major detectors at RHIC, only STAR has the large acceptance required for detection of jets produced in $p p$ collisions. The central element of STAR is the time projection chamber, providing charged particle tracking through a $0.5 \mathrm{~T}$ magnetic field over the pseudorapidity range, $|\eta|<2$. The barrel electromagnetic calorimeter will provide good measurements of photons and electrons at mid-rapidity $(|\eta|<1)$.

The primary objective of the STAR-spin effort is the measurement of the fraction of the proton's spin carried by gluons, given by the first moment of the polarized gluon structure function, $\Delta G\left(x_{g}\right)$, where $x_{g}$ is the Bjorken variable. By far, the cleanest probe of $\Delta G\left(x_{g}\right)$ is direct photon production, since the process is dominated by gluon Compton scattering. Coincidence detection of the away-side jet enables an event-by-event determination of the initial-state parton kinematics. By focusing on collisions where $x_{q}>0.2$, it is possible to restrict attention to events where the quark has a large and well known polarization, $A_{p}^{1}\left(x_{q}\right)$, measured in polarized deep inelastic scattering. Since the bulk of the gluons are expected at small $x_{g}$, this suggests the need to study asymmetric partonic collisions. As well, the partonic two-spin asymmetry $\left(\hat{a}_{L L}\right)$ is maximal when the photon is detected in the direction of the incident quark. These two features suggest that the addition of an endcap electromagnetic calorimeter (EEMC) to STAR (Fig. 1) will optimize the detector for determining the integral $\Delta G$.

Experimentally, direct photon detection is challenging because of the large flux of energetic neutral mesons $\left(\pi^{0}, \eta^{0}\right)$ that most probably decay into photon pairs with small opening angles. As shown in Fig. 2, isolation cuts are a powerful means of suppressing the direct meson background. The design of the EEMC has been optimized to detect high transverse momentum photons $\left(p_{T}>10 \mathrm{GeV} / \mathrm{c}\right)$ at large pseudorapidity, $1 \leq \eta \leq 2$. A crucial element is the shower maximum detector, used to measure the transverse profile of electromagnetic showers thereby providing the capability to reconstruct direct meson events and further suppress the background.

Neglecting the small contribution from $q \bar{q}$ annihilation, the asymmetry for direct photon detection is particularly simple in leading order perturbative QCD:

$$
A_{L L}=\frac{\Delta G\left(x_{g}\right)}{G\left(x_{g}\right)} A_{p}^{1}\left(x_{q}\right) \hat{a}_{L L} .
$$

Since the kinematics of the initial state partons can be reconstructed for each event, this simple expression can be inverted to provide a direct extraction of $\Delta G(x)$ from the measured asymmetries as shown in Fig. 3. As shown in Fig. 4, extrapolation errors will limit the determination of the integral $\Delta G$ based on the analysis of $200 \mathrm{GeV}$ direct photon data, alone. The addition of a data set at $\sqrt{ } s=500 \mathrm{GeV}$ will extend the $x_{g}$ range to the lower limit of 0.01 , thereby reducing the extrapolation errors by a factor of five. Overall, the systematic error estimate is that the integral $\Delta G$ can be determined to \pm 0.5 at STAR with the EEMC addition.

The STAR spin program is summarized in Fig. 5. More details about the program are contained in a proposal for the EEMC addition to the BNL program advisory committee, available as a link at the URL http://www.rhic.bnl.gov/STAR/html/spin_l/ 


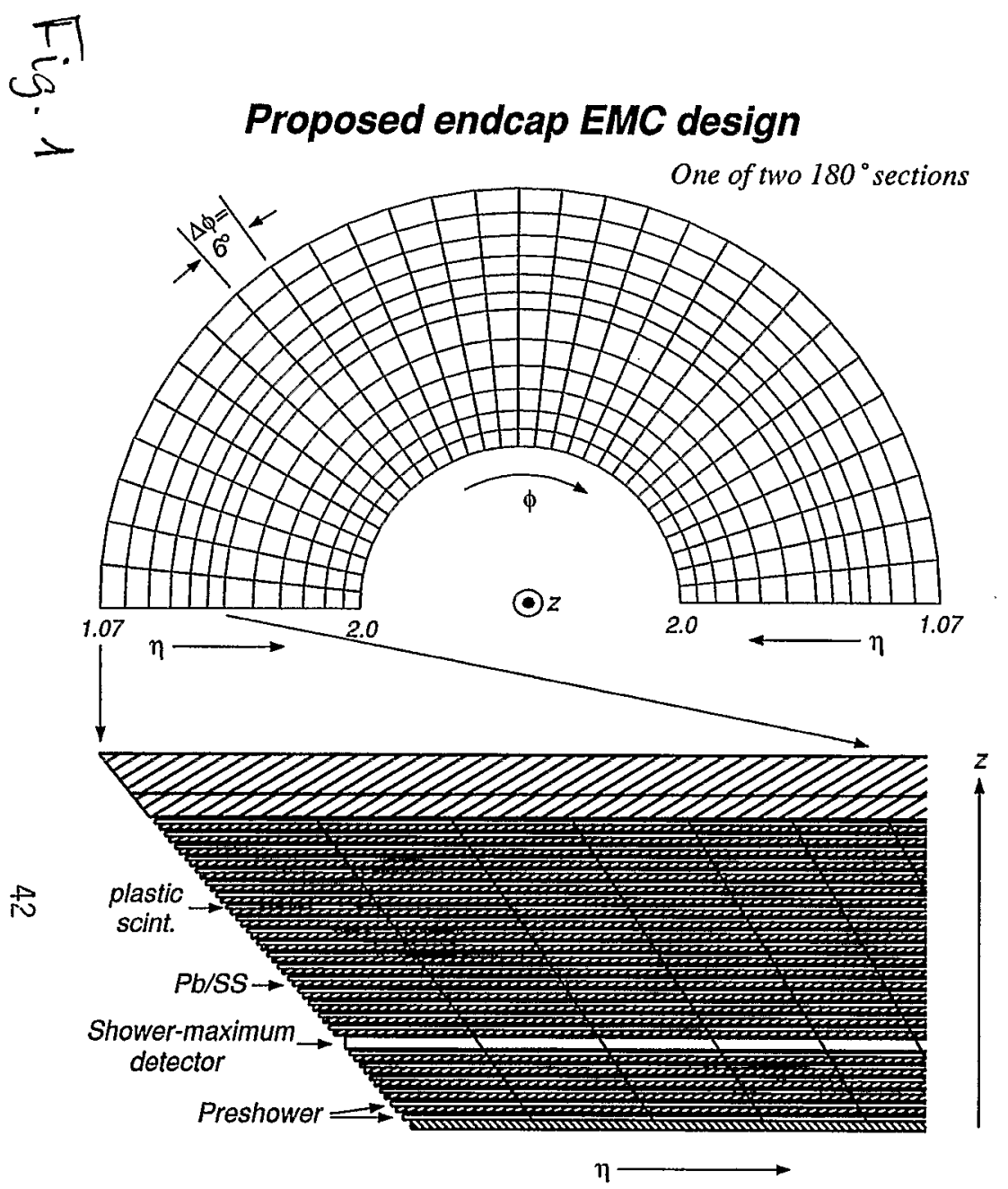

Summary of Design Features

- total of 720 projective "towers"

- for azimuthal coverage for pseudorapidity interval $1.07 \leq \eta \leq 2.00$

- tower size $(\Delta \eta, \Delta \phi)=(0.05,0.1)$ for $1.07<\eta<1.37$

$$
(0.1,0.1) \text { for } 1.37<\eta<2.00
$$

- $P b$ - scintillator sampling calorimeter of depth $\sim 21$ radiation length (normal incidence)

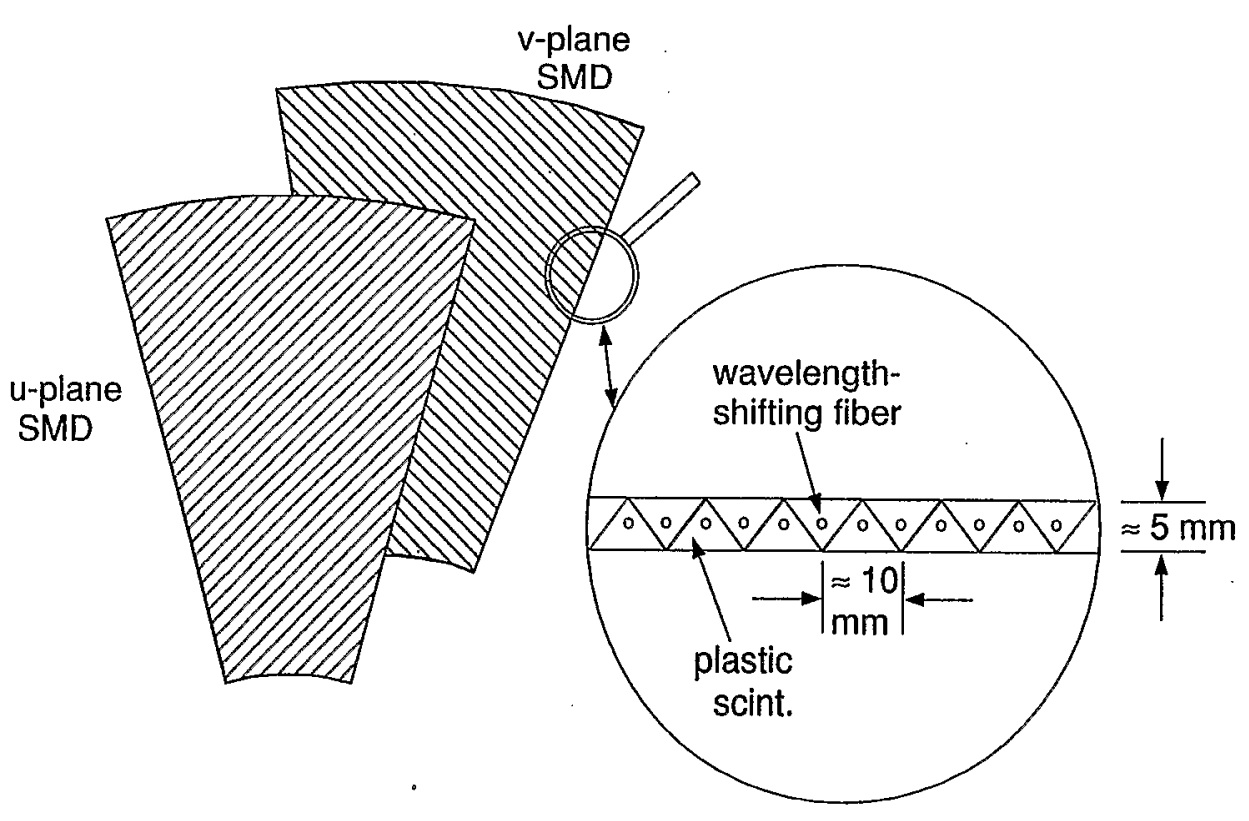

\section{Proposed Shower Maximum Detector (SMD) design}

The SMD detector consists of triangular cross section scintillator strips (designed for the DO upgrade) and is used to distinguish a single direct photon from a pair of photons from $\pi^{0}$ decay by measuring the transverse profile of an electromagnetic shower. 


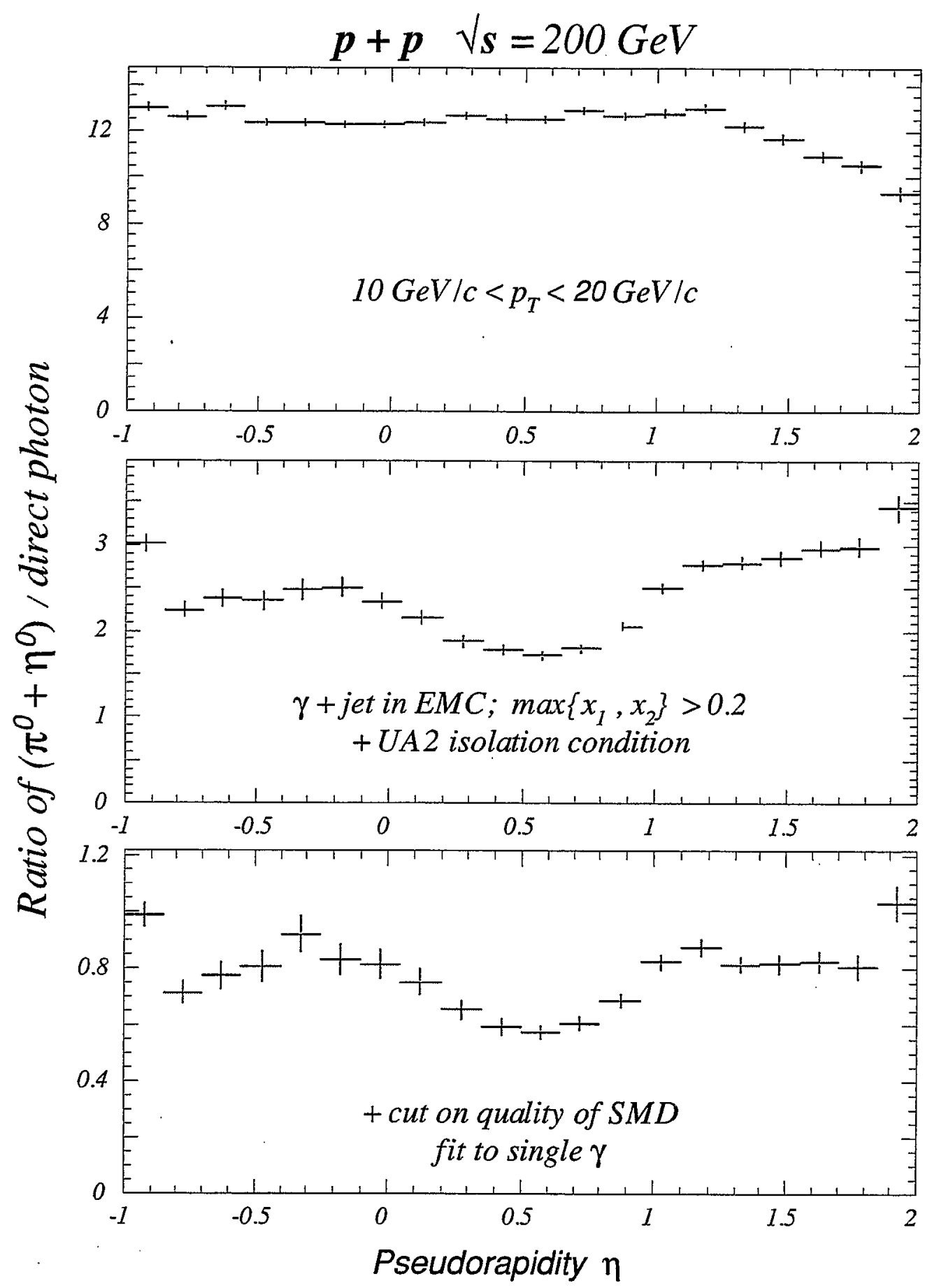

Fig. 2

The cross section ratio of $\pi^{0}\left(\eta^{0}\right)$ meson production to direct photon production in pp collisions at $\sqrt{s}_{s}=200 \mathrm{GeV}$, generated with PYTHIA 5.7, with the following conditions imposed: (Top) limiting only the transverse momentum to $10 \leq p_{T} \leq 20 \mathrm{GeV} / \mathrm{c}$; (Middle) after application of conditions including the UA2 isolation cut; (Bottom) after additional application of direct meson suppression based on measurements from the SMD. For these simulations, the SMD was placed after the fifth layer of the calorimeter 


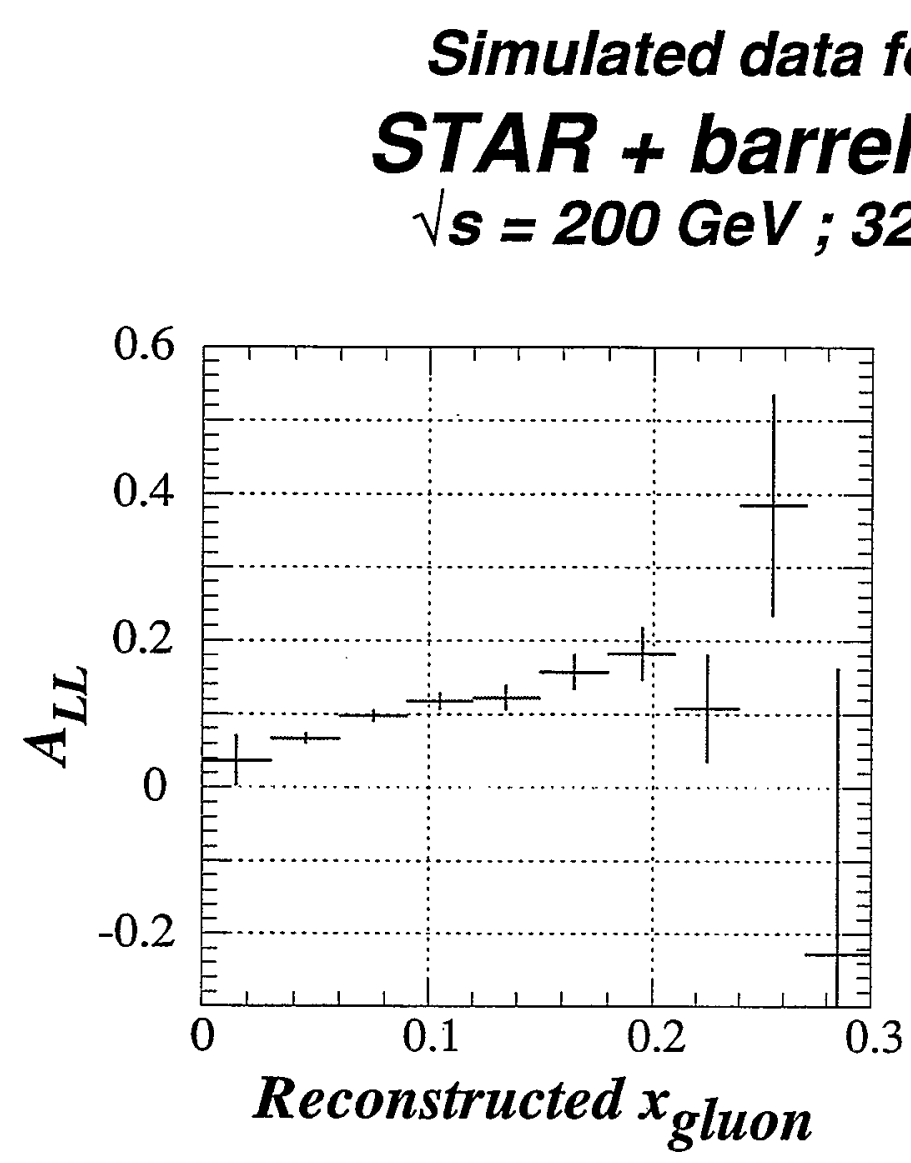

- error bars are statistical only

- remnant $\pi^{0}$ background subtraction increases statistical errors by factor $\approx 1.5-2.0$

- \pm 0.005 systematic error due to instrumental asymmetry $\Rightarrow \pm 0.010$ error in $A_{L L}$

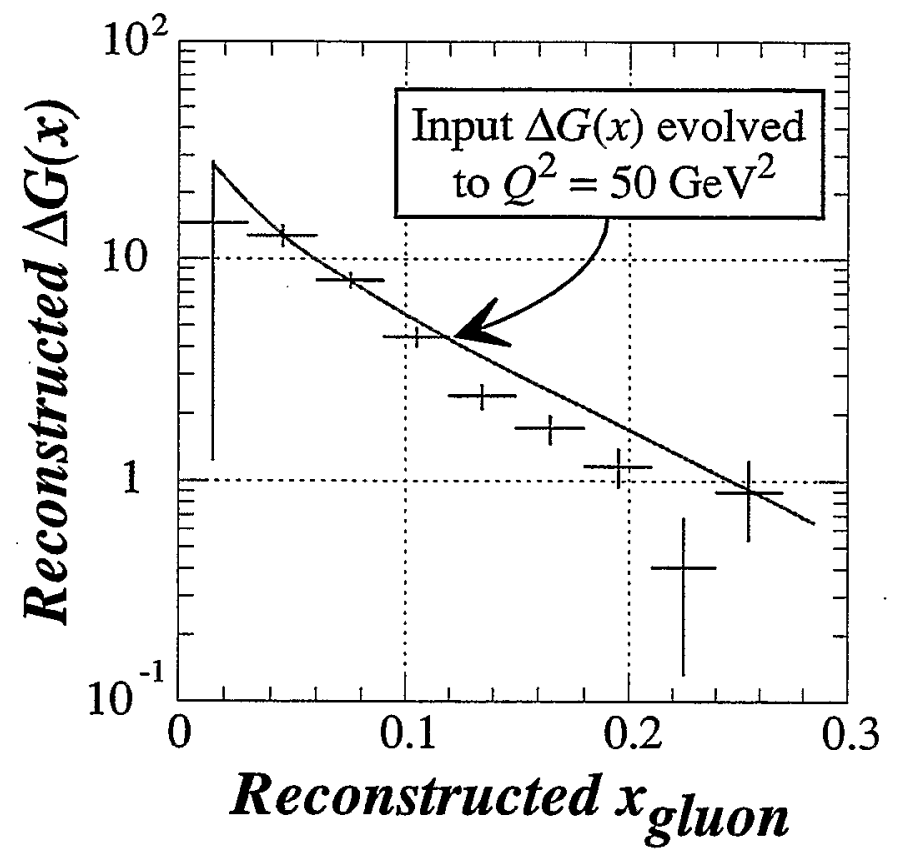

Systematic deviation from input $\triangle G\left(x, Q^{2}\right)$ arise from neglect of:

- $q \bar{q} \rightarrow \gamma g$

- $x_{\text {gluon }} \leftrightarrow x_{\text {quark }}$ ambiguity

- $\cos \vartheta^{*}$ resolution

- $k_{T}$ smearing

All effects tend to reduce reconstructed $\Delta G(x)$. Effects $\rightarrow 0$ for $x_{\text {gluon }}<0.1$ where contributions to $\int_{0}^{1} \Delta G(x) d x$ are largest

$\mathrm{Fg} \cdot 3$ 
Attempt to fit reconstructed $\Delta G(x)$ from $v_{s}=200 \mathrm{GeV}$ "measurements" with Gehrmann \& Stirling function:

$x \Delta G(x)=\eta_{G} A_{G} x^{a_{G}}(1-x)^{b_{G}}\left(1+\gamma_{G} x+\rho_{G} x^{1 / 2}\right)$

$A_{G}$ chosen so $\int_{0}^{1} \Delta G(x) d x=\eta_{G}$ fixed parameters

varied parameters

$$
\begin{aligned}
& b_{G}=\mathbf{5 . 7 1} \\
& \gamma_{G}=\mathbf{0}
\end{aligned}
$$

$\eta_{G}$

$a_{G}$

$\rho_{G}$

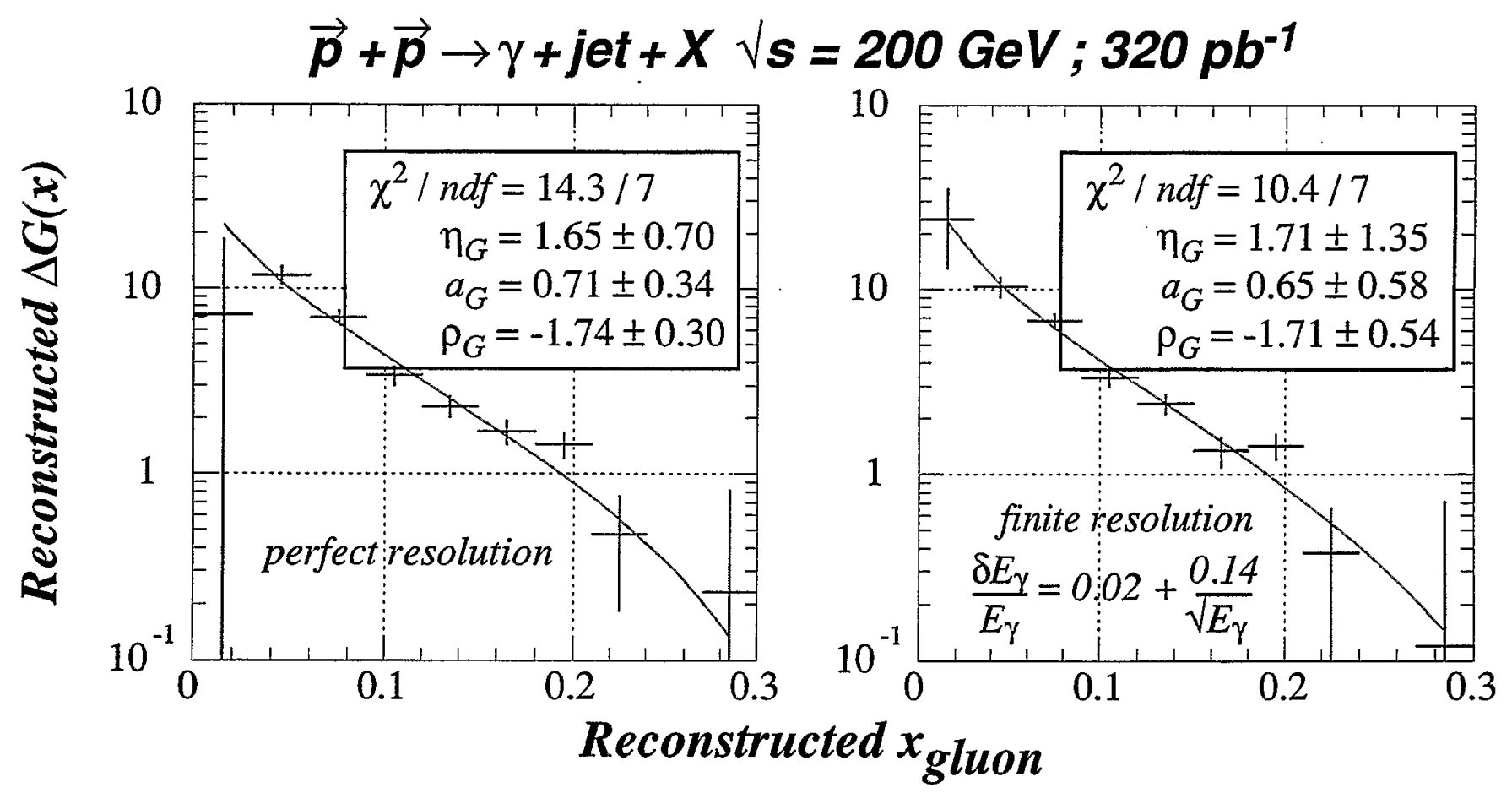

$\Rightarrow$ strong correlation between $\boldsymbol{a}_{G}$ and $\eta_{G}$

$\Rightarrow$ significant extrapolation errors fitting $\sqrt{ }_{s}=200 \mathrm{GeV}$ data only

Fig. 4 


\section{Summary of STAR-spin Program}

1) Determination of the integral $\Delta G$ via measurements of two-spin asymmetries $\left(A_{L L}\right)$ for the

$$
\vec{p}+\vec{p} \rightarrow \gamma+\text { jet }+X
$$

reaction at $\sqrt{ } s=200$ and $500 \mathrm{GeV}$ (two $\sim 10$-week runs).

2) Measurement of two-spin asymmetries $\left(A_{L L}\right)$ for

$$
\vec{p}+\vec{p} \rightarrow d i-j e t+X
$$

$\Rightarrow$ double check $\Delta G(x)$ extracted from $\gamma+$ jet data.

3) Determination of sea-quark polarized structure functions ( $\Delta \bar{u}$ and $\Delta \bar{d}$ ) by measurement of parity-violating longitudinal spin asymmetries in

$$
\vec{p}+p \rightarrow W^{ \pm}+X \rightarrow e^{ \pm}+(v)+X
$$

4) Other possible measurements...

- information about polarized fragmentation functions

$$
\vec{p}+p \rightarrow \vec{Y}+X
$$

- parity violation in di-jet production

$\Rightarrow$ physics beyond the Standard Model

Fig. 5 


\title{
PHENIX Spin Physics
}

\author{
N. HAYASHI
}

RIKEN,

The Institute of Physical

and Chemical Research

Sep 22. 1998

Workshop on Event Generator for RHIC Spin

Physics,

RIKEN BNL Reserch Center 


\section{PHENIX Spin Physics}

\section{N.HAYASHI}

The spin-dependent structure functions of the nucleon, particularly the quark spin contributions, have been studied extensively in polarized deep inelastic scattering (pDIS) experiments in the last decade. However, the the gluon spin contribution to the nucleon spin remains as a weakly constrained quantity. Moreover, pDIS inclusive reaction alone is not possible to separate individual contributions of each quark flavor or that of the anti-quark. RHIC/Spin experiments are the new type of the experiments which use polarized proton-proton collisions at very high energy.

We discuss measurements of the gluon polarization via the prompt photon, $\pi^{0}$, quarkonium and open heavy quark production at PHENIX. Double longitudinal asymmetry $A_{L L}$ can be expressed by two parton distribution functions (PDF) and subprocess asymmetry $\hat{a}_{L L}$. Since $A_{L L}$ is going to be measured, and $\hat{a}_{L L}$ is calculable, one can extract the polarized PDF. Some theoretical uncertainties has raised but the prompt photon is still one of the best reaction to get $\Delta G$ information. $\pi^{0}$ has model dependent part, but its extremely high statistics is very attracting. Quarkonium production, particularly $J / \psi$, is interested in both heavy ion physics and spin physics. Large fraction of the quarkonium or the open heavy flavor particle come from $g+g$ or $g+q$, so it is useful to study the gluon structure. Unfortunately, its production mechanism is not settled yet completely. Open heavy flavor signal can be detected with high $p_{T}$ single muon.

The production of $W$ is quite interesting and it is described how to disentangle the contribution from different (anti-) quark flavor via $W$ production. In terms of anti-quark measurement, Drell-Yan process is an interesting one, but the heavy quark semi-leptonic decay seems to be high background source. It is still under study.

For beyond the structure function study, there are other interesting quantities, the transverse single spin asymmetry $A_{N}$, which is related to higher twist effect, or $A_{T T}$, is related to the transversity and parity violation may be a key for beyond standard model physics. 
Gluon Helicity Distribution: $\Delta g$

\begin{tabular}{lccr}
\hline \hline asymmetry & measure & $\sqrt{s}$ & detector required* \\
\hline $\mathcal{A}_{L L}(p p \rightarrow \gamma X)$ & $\Delta g \times g_{1}$ & $200 / 500 \mathrm{GeV}$ & EMCal+Trk \\
$\mathcal{A}_{L L}(p p \rightarrow c \bar{c} X)$ & $\Delta g \times \Delta g$ & $200 \mathrm{GeV}$ & Muon+EMCal \\
$\mathcal{A}_{L L}(p p \rightarrow b \bar{b} X)$ & $\Delta g \times \Delta g$ & $500 \mathrm{GeV}$ & Muon+EMCal \\
$\mathcal{A}_{L L}\left(p p \rightarrow \pi^{0} X\right)$ & $(\Delta g)^{2} / \Delta g \times \Delta q$ & $200 / 500 \mathrm{GeV}$ & EMCal \\
\hline \hline
\end{tabular}

Anti-quark Helicity Distribution: $\Delta \bar{q}$

\begin{tabular}{lccc}
\hline \hline asymmetry & measure & $\sqrt{s}$ & detector required $^{*}$ \\
\hline $\mathcal{A}_{L L}\left(p p \rightarrow \gamma^{*} X\right)$ & $\Delta q \times \Delta \bar{q}$ & $50(?) \mathrm{GeV}$ & Muon \\
$\mathcal{A}_{L}\left(p p \rightarrow W^{+} X\right)$ & $\Delta u, \Delta \bar{d}$ & $500 \mathrm{GeV}$ & Muon/EMCal \\
$\mathcal{A}_{L}\left(p p \rightarrow W^{-} X\right)$ & $\Delta d, \Delta \bar{u}$ & $500 \mathrm{GeV}$ & Muon/EMCaI \\
\hline \hline
\end{tabular}

Flavor Decomposition of Quark/Anti-quark Helicity Distribution: $\Delta q_{i} / \Delta \bar{q}_{i}$

\begin{tabular}{lccc}
\hline \hline asymmetry & measure & $\sqrt{s}$ & detector required* \\
\hline $\mathcal{A}_{L}\left(p p \rightarrow W^{+} X\right)$ & $\Delta u, \Delta \bar{d}$ & $500 \mathrm{GeV}$ & Muon/EMCal \\
$\mathcal{A}_{L}\left(p p \rightarrow W^{-} X\right)$ & $\Delta d, \Delta \bar{u}$ & $500 \mathrm{GeV}$ & Muon/EMCal \\
\hline \hline
\end{tabular}

Quark/Anti-quark Transversity Distribution: $\delta \boldsymbol{q} / \delta \bar{q}$

\begin{tabular}{lccc}
\hline \hline asymmetry & measure & $\sqrt{s}$ & detector required \\
\hline $\mathcal{A}_{T T}\left(p p \rightarrow \gamma^{*} X\right)$ & $\delta q \times \delta \bar{q}$ & $50-500 \mathrm{GeV}$ & Muon \\
\hline \hline
\end{tabular}

Parity Violation in Standard Model and in Beyond SM

\begin{tabular}{lccr}
\hline \hline asymmetry & measure & $\sqrt{s}$ & detector required* \\
\hline $\mathcal{A}_{L}(p p \rightarrow$ jet $X)$ & compositeness scale & $500 \mathrm{GeV}$ & EMCal+Trk \\
$\mathcal{A}_{L}\left(p p \rightarrow \gamma^{*} / Z X\right)$ & $\gamma / Z$ interference & $500 \mathrm{GeV}$ & Muon \\
\hline \hline
\end{tabular}

\section{Higher twist effects in Polarization Phenomena}

\begin{tabular}{lccr}
\hline \hline asymmetry & measure & $\sqrt{s}$ & detector required* \\
\hline $\mathcal{A}_{N}(p p \rightarrow \gamma X)$ & higher-twist & $200 / 500 \mathrm{GeV}$ & EMCal+Trk \\
$\mathcal{A}_{N}\left(p p \rightarrow \gamma^{*} X\right)$ & higher-twist & $50-500 \mathrm{GeV}$ & Muon \\
$\mathcal{A}_{N}\left(p p \rightarrow \pi^{0} X\right)$ & higher-twist & $200 / 500 \mathrm{GeV}$ & EMCal \\
\hline \hline
\end{tabular}

TABLE II. Spin physics with PHENIX detector system. In the column of "detector required", BB and MVD are always assumed. 


\section{Prompt Photon Asymmetry}

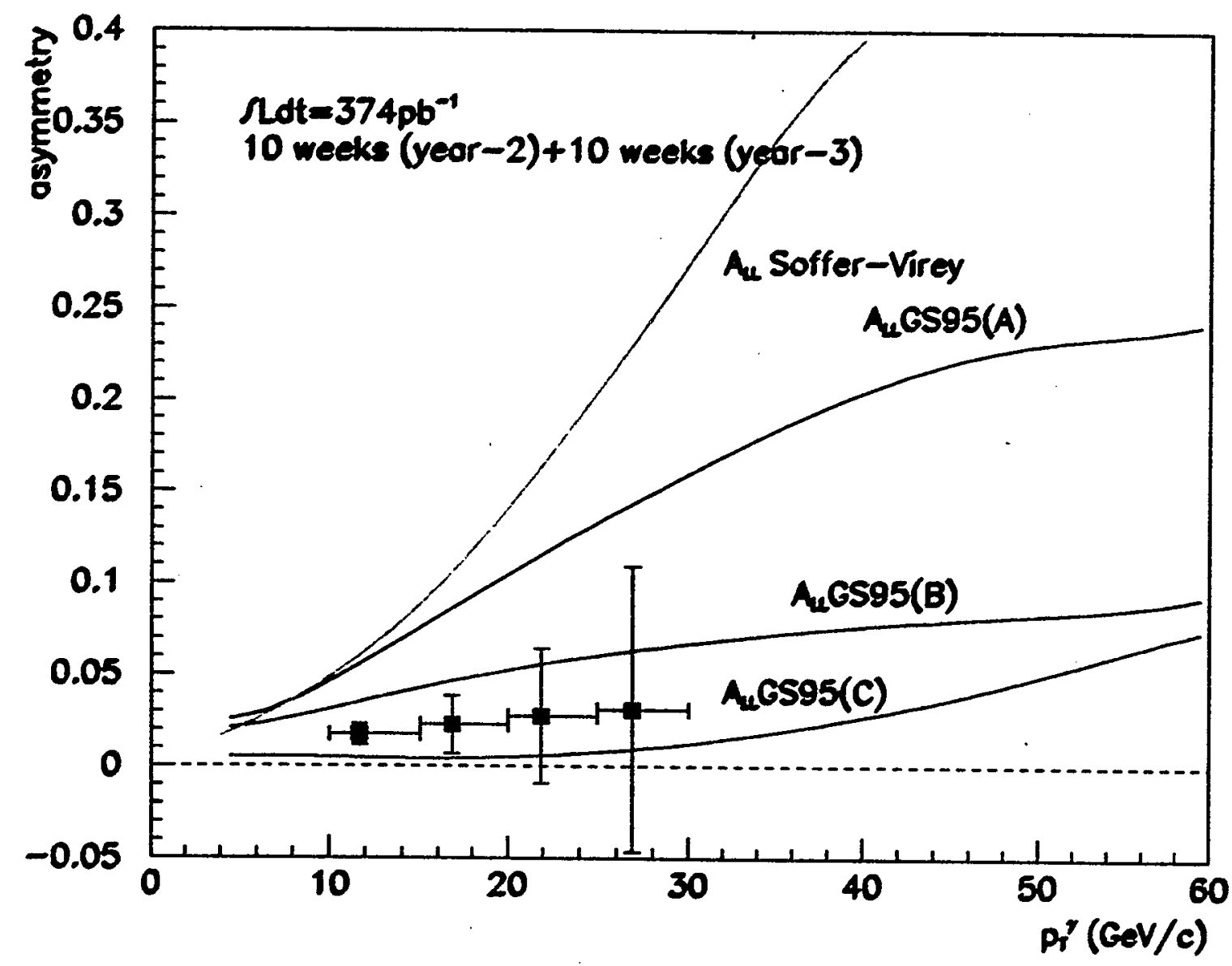


- $\pi^{0}$ measurement

\section{- luminosity}

- year-2 $-34 \mathrm{pb}^{-1}$ for $\mathrm{sqrt}(\mathrm{s})=200 \mathrm{GeV}$

- yield

\begin{tabular}{c|r|r}
\hline \hline \multirow{2}{*}{$\pi^{0} p_{T}$} & \multicolumn{2}{|c}{$\sqrt{\mathrm{s}=200 \mathrm{GeV}}$} \\
\cline { 2 - 3 } $2-3 \mathrm{GeV} / \mathrm{c}$ & $3.3 \times 10^{8}$ & $1.1 \times 10^{-4}$ \\
$3-4 \mathrm{GeV} / \mathrm{c}$ & $4.3 \times 10^{7}$ & $3.1 \times 10^{-4}$ \\
$4-5 \mathrm{GeV} / \mathrm{c}$ & $8.8 \times 10^{5}$ & $6.9 \times 10^{-4}$ \\
$5-6 \mathrm{GeV} / \mathrm{c}$ & $2.3 \times 10^{6}$ & $1.3 \times 10^{-3}$ \\
$6-7 \mathrm{GeV} / \mathrm{c}$ & $7.4 \times 10^{5}$ & $2.4 \times 10^{-3}$ \\
$7-8 \mathrm{GeV} / \mathrm{c}$ & $3.5 \times 10^{5}$ & $3.4 \times 10^{-3}$ \\
$8-9 \mathrm{GeV} / \mathrm{c}$ & $1.3 \times 10^{5}$ & $5.6 \times 10^{-3}$ \\
$9-10 \mathrm{GeV} / \mathrm{c}$ & $6.8 \times 10^{4}$ & $7.8 \times 10^{-3}$ \\
$10-11 \mathrm{GeV} / \mathrm{c}$ & $3.1 \times 10^{4}$ & $1.2 \times 10^{-2}$ \\
$11-12 \mathrm{GeV} / \mathrm{c}$ & $2.1 \times 10^{4}$ & $1.4 \times 10^{-2}$ \\
\hline \hline
\end{tabular}

Table 1: $\pi^{0}$ yield

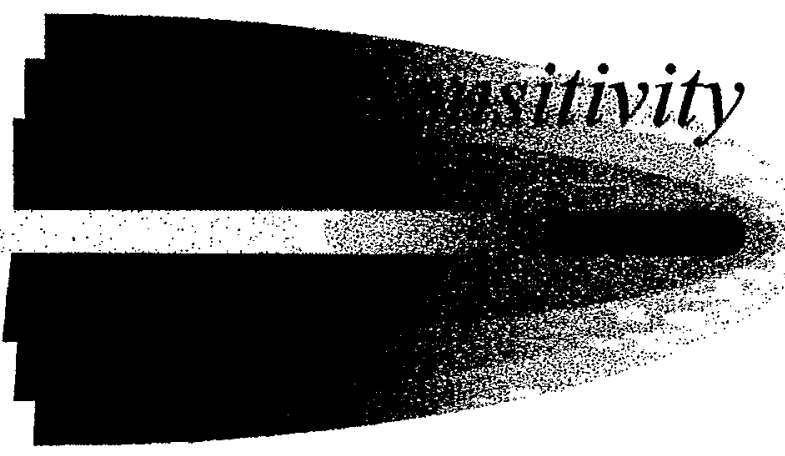

- invariant mass $-5 \mathrm{Gev} / \mathrm{c} \mathrm{p}_{\mathrm{T}}$ cut

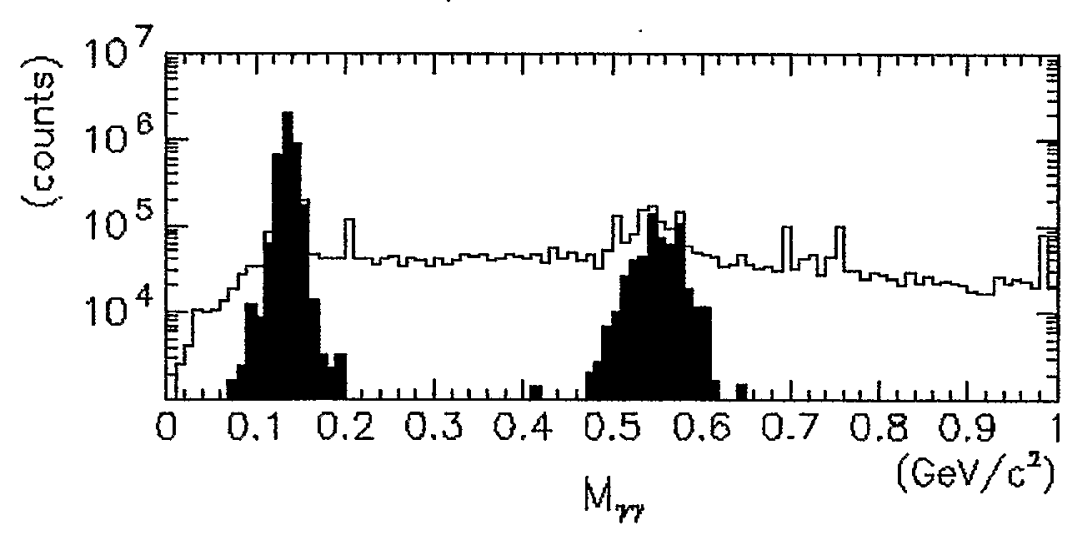




\section{Sensitivity}

statistical limit

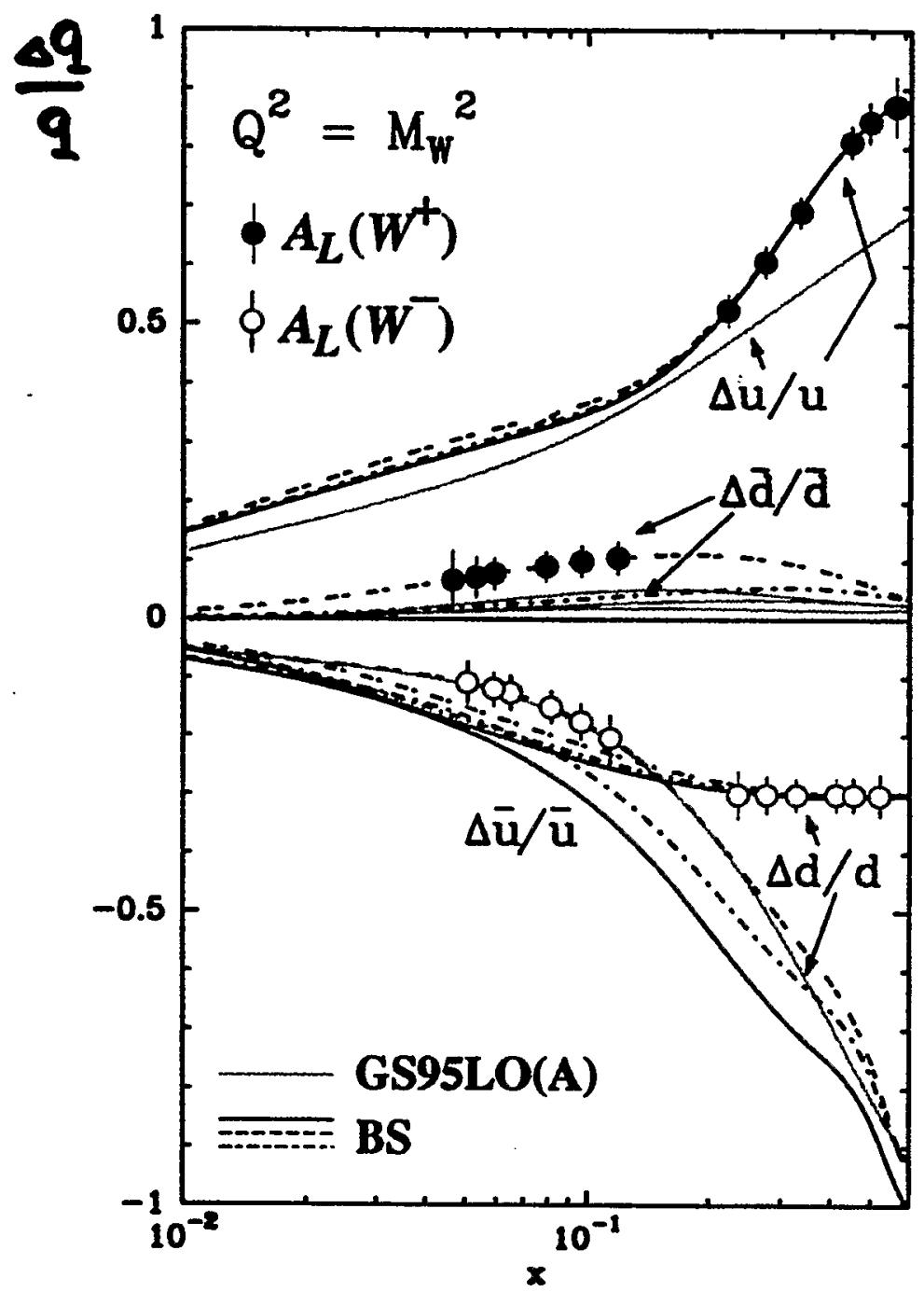

courtey ef Jacques Soffer \& Cloude Bourrely

- Anti-quark polarization measured with $A_{L}^{W}$

- More systematic studies are underway

- background from $\pi / K$ decays

- background from $Z^{0}$ decays 


\section{Summary}

- Spin Structure Function Studies

- $\Delta G(x)$ measurements:

* prompt $\gamma^{*} 5-30 \%$ at $\sqrt{s}=200 \mathrm{GeV}, 0.1<x<0.3$

$5-20 \%$ at $\sqrt{s}=500 \mathrm{GeV}, 0.04<x<0.16$

* inclusive $\pi^{0}-$ Model dependent, but high statistics

* quarkonium production - more understanding of production mechanism needed

* open heavy quark - NLO calculation .

- $\Delta \bar{q}(x)$ measurements and flavor decomposition:

* $2 \%$ error through $A_{L}^{W}$

* Drell-Yan process - background from semi-leptonic decay need study

- More Topics

- Single Transverse Spin Asymmetry

- Transversity Measurement - Pol.DY, Two mesons

- Parity Violation 


\title{
Polarized Parton Distributions and Event Generator .
}

\author{
T.-A. Shibata \\ Tokyo Institute of Technology \\ shibata@nucl.phys.titech.ac.jp
}

September 23, 1998

One of the major goals of the RHIC SPIN experiments is to determine the polarized parton distributions as a function of $x$ and $Q^{2}$ to understand the nucleon structure in terms of QCD. To this end I report what we have obtained up to now and what we plan in future.

1. Polarized parton distribution functions were obtained with a new parametrization using world data from the longitudinally polarized deep inelastic leptonnucleon scattering experiments. A program of $Q^{2}$ evolution up to Vext-toLeading order was developped in order to use it in the fit. A new parametrization of the parton distribution functions was then adopted taking into account the positivity, helicity retention etc. From the fit of the $A_{1}$ data, the polarizded distribution functions of $u$ and $d$ valence quarks, sea quarks, and gluon were determined. The errors in the parameter determination were evaluated. The first moments were obtained.

2. The mock data of RHIC SPIN can be used as input to the fit in the procedure described above. We can then test how well RHIC data will determine the polarized parton distributions. If necessary, a combination of the mock data from RHIC and the data from lepton-nucleon deep inelastic scattering can be used as input.

3. The polarized parton distibutions described above can be used as input to the Event Generator. By changing the input functions we will test the sensitivity of RHIC experiments (acceptance, energy resolution etc.) to the polarized parton distributions. By analysing the Vonte Carlo data we will test how well we can extract back the input functions. 
Activities around RIKEN

RHIC SPIN J Physics Discussion

- monthly

+ Working Group on Polarized Parton Distribution Functions

- fit the World Data and publish

- predictions for RHIC SPIN

RIKEN Symposium on 'Quarks and Gluons in the Nucleon' Nov 1997, at RIKEN

RIKEN Winter School 'Structure of Hadrons

- Introduction to QCD Hard Process'

Dec 9-12, 1998, at Shimoda in Japan

Lecturers : R. Safe, K. Roth, J. Kodaira

55 
One of the main goals of RHIC SPIN is to determine polarized parton distribution functions by its own data, or by its own data + lepton D.I.S data

Discriminating between the existing parametrizations (model $1,2,3 \ldots)$ is not the ultimate goal. ex.

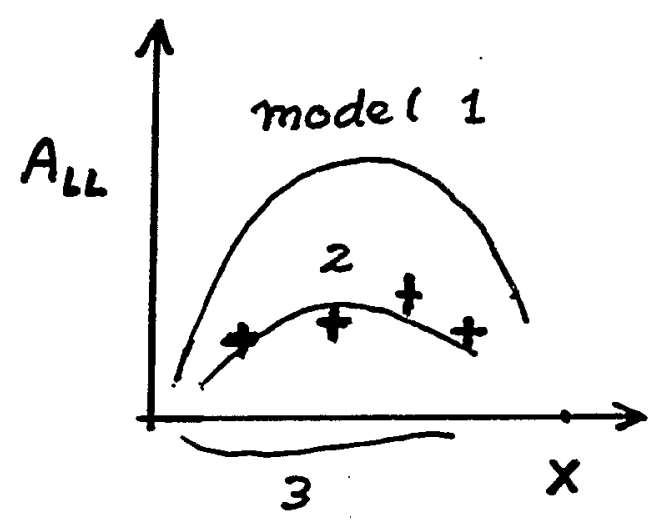

many-to- one correspondence

56 
NLO fit of polarized parton distribution functions

RHIC SPIN

(1) pol.

D. I. S data Mock Data Real Data

A

$A_{L L}, \cdots$

$\downarrow$

$\downarrow$

parametrization $Q^{2}$ evolution

$f_{i t}: \quad x^{2}=\sum_{i} \frac{\left(f\left(x, Q^{2}\right)-\text { data }_{i}\right)^{2}}{\sigma_{i}^{2}}$

$\Downarrow$

$\Delta u, \Delta d, \Delta \bar{u}, \Delta \bar{d}, \Delta s, \Delta \bar{s}$

$\Delta g \quad\left(X, Q^{2}\right)$

To check:

How much RHIC SPIN data will contribute to determine pol. PDF's.

57 
(2). Monte Carlo Simulation

$$
\Delta u, \Delta d, \Delta \bar{u}, \Delta \bar{d}, \Delta S, \Delta \bar{s}, \Delta g\left(x, Q^{2}\right)
$$

$\Downarrow$

Event Generator

$\sqrt{1}$ $\left.\left.\begin{array}{l}\text { (digitization), acceptance } \\ \text { efficiency, energy-. } \\ \text { angle-resolutions }\end{array}\right\} \begin{array}{l}\text { simplified version } \\ \text { of detector response }\end{array}\right\}$

- Test of Sensitivity of RHIC SPIN experiments to pol. PDF's.

- Extract back the input $\Delta g\left(x, Q^{2}\right)$ etc. from $M C$ data

Test of the overall precision of experimem one-to-one correspondence?
(input) (output) uniquely determined?

58 
Summary of the fit

With NLO analysis of the polarized parton distribution functions $\quad X=198.30 / 220$ for NLO, $x^{2}=246.42 / 220$ for LO were obtained.

$\Delta g$ is poorly determined. A new direct data on $\Delta g\left(X, Q^{2}\right)$ which discriminates between $\Delta g=1.9$ and 0.7 with $x^{2}$ increase of 0.7 will have the same impact as the whole pol. D.I.S data in determining $\Delta g$.

59 


\section{An Introduction to Resummation and Intrinsic $p_{T}$ \\ George Sterman, Institute for Theoretical Physics, SUNY Stony Brook}

The influence of partonic transverse momentum on single-particle inclusive cross sections has been a subject of interest for some time. The standard factorized cross section for $A+B \rightarrow$ $C+X$ is a convolution in momentum fractions only,

$$
E_{C} \frac{d \sigma_{A B \rightarrow C+X}}{d^{3} p_{C}}=\sum_{a b c} f_{a / A}\left(x_{a}, \mu^{2}\right) \otimes f_{b / B}\left(x_{b}, \mu^{2}\right) \otimes D_{C / c}\left(z, \mu^{2}\right) \otimes \hat{\sigma}\left(x_{a} p_{A}, x_{b} p_{B}, p_{C} / z, \mu\right) .
$$

Transverse momenta $k_{T}<\mu$ (relative to the incoming and outgoing directions) are absorbed into parton distributions $f$ and fragmentation functions $D$, while larger transverse momenta are included in the partonic hard scattering $\hat{\sigma}$. Corrections associated with this organization of states appear systematically in higher-order corrections to $\hat{\sigma}$. An important example is direct photon production, which is of special relevance to the determination of the gluon distribution. Data on direct, or prompt, photons is available over a wide range of $\sqrt{s}$ and $p_{T}$, generally with isolation cuts at collider energies. For some time, many, although not all [1], calculations of direct photon production based on Eq. (1) have fallen significantly below the data at low $p_{T}$, suggesting the need for a " $k_{T}$-smearing", perhaps reflecting the intrinsic transverse momentum of partons in hadrons [2].

An alternative factorization including transverse momenta is [3]

$$
\begin{aligned}
E_{\ell} \frac{d \sigma_{A B \rightarrow \gamma(\ell)+X}}{d^{3} \ell}=\frac{1}{S^{2}} & \sum_{a b} \int d x d y d^{2} \mathbf{q} d^{2} \mathbf{q}^{\prime} \mathcal{P}_{a / A}\left(x, p_{A} \cdot n, \mathbf{q}\right) \mathcal{P}_{b / B}\left(y, p_{B} \cdot n, \mathbf{q}^{\prime}\right) \\
& \times \Omega_{a b \gamma}\left(\frac{s^{\prime}}{\mu^{2}}, \frac{t^{\prime}}{\mu^{2}}, \frac{u^{\prime}}{\mu^{2}}, \alpha_{s}\left(\mu^{2}\right)\right)
\end{aligned}
$$

with a modified hard-scattering function $\Omega$, which depends on kinematic variables $s^{\prime}, t^{\prime}, u^{\prime}$ that include parton transverse momenta. The wave functions $\mathcal{P}$ are related to the light-cone wave functions $f$ in Eq. (1) by

$$
\mathcal{P}_{a / A}(x, p \cdot n, \mathbf{q})=\sum_{c} \int d \lambda f_{c / A}\left(\lambda, \mu^{2}\right) \int d^{2} \mathbf{b} e^{-i \mathbf{q} \cdot \mathbf{b}} C_{a / c}\left(\frac{x}{\lambda},|\mathbf{b}| \mu, \alpha_{s}\left(\mu^{2}\right)\right) \mathrm{e}^{-S_{c}(|\mathbf{b}|, p \cdot n)},
$$

where the $b$-dependent exponent, which matches perturbative and nonperturbative contributions [4], has the effect of introducing a Gaussian $k_{T}$-smearing into the cross section (2). $n^{\mu}$ is an arbitrary gauge-fixing vector, so that $p \cdot n$ increases with the center-of-mass energy. The exponent $S$ produces a $k_{T}$-smearing with a width that increases like the logarithm of $s$, as suggested by the analyses of [2].

\section{References}

[1] P. Aurenche et al., Phys. Rev. D39, 3275 (1989).

[2] J.F. Owens, Rev. Mod. Phys. 59, 465 (1987); J. Huston et al., Phys. Rev. D51, 6139 (1995); H. Baer and M.H. Reno, Phys., Rev. D54, 2017 (1996); A.D. Martin et al, hep-ph/9803445.

[3] H.-L. Lai and H.-n. Li, hep-ph/9802414; E. Laenen, G. Oderda and G. Sterman, in preparation.

[4] J.C. Collins and D.E. Soper, Nucl. Phys. B193, 381 (1981). J.C. Collins, D.E. Soper and G. Sterman, Nucl. Phys. B250, 199 (1985). 
Direct (= Prompt) Photons

$$
\begin{aligned}
A+B & \rightarrow \gamma(l)+X \\
E_{l} \frac{d^{3} \sigma}{d l^{3}}= & \int d x_{a} d x_{b} f_{a / A}\left(x_{a}\right) f_{b / B}\left(x_{b}\right) \\
\cdot \hat{\sigma}_{a+b \rightarrow \gamma+X}\left(x_{a} P_{A}, x_{b} P_{B}, l, \mu^{2}\right) & +f_{\text {fragmentation }} \\
& \text { corrections }
\end{aligned}
$$

- Fragmentation at NLO...

for high lo, cut down further by isolation

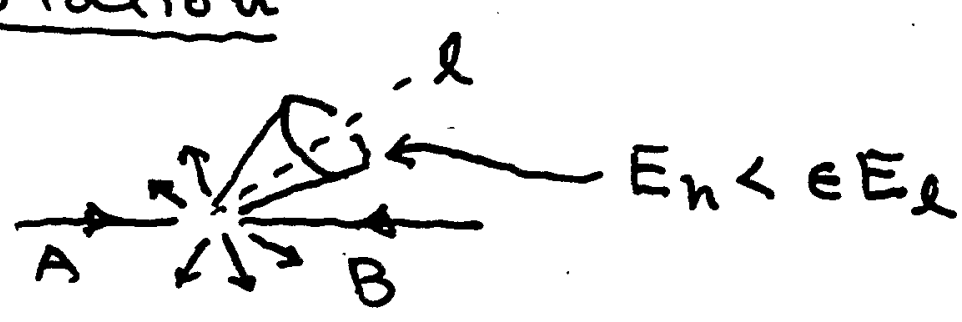

- Gluon distribution enters at LO:

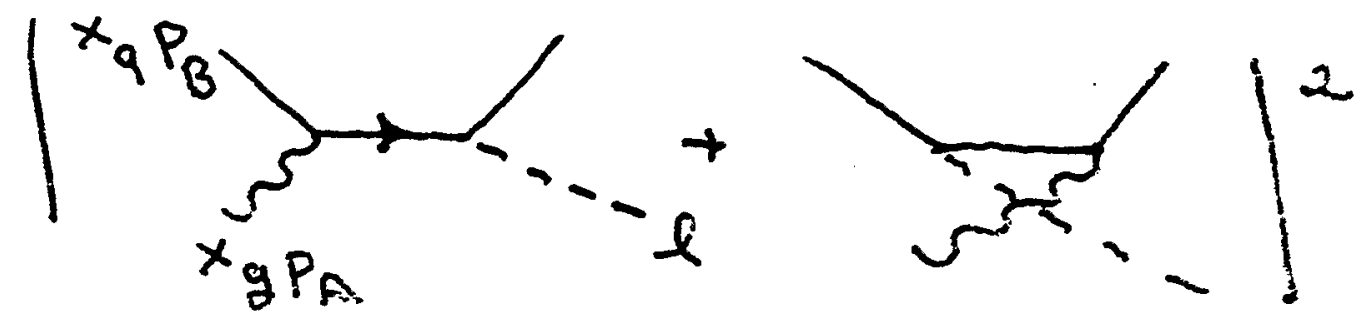

- Data for wide range of $l_{T}, \sqrt{5}$

- Status of NLO...

61 
CTEQ' Analysis:

J. Huston, E. Kovacs, S. Kuhlmann, H.L.Lai, J.F. Owens, W.K. Tong Phys. Rev. D51,6139(1995) hep-ph!9501230

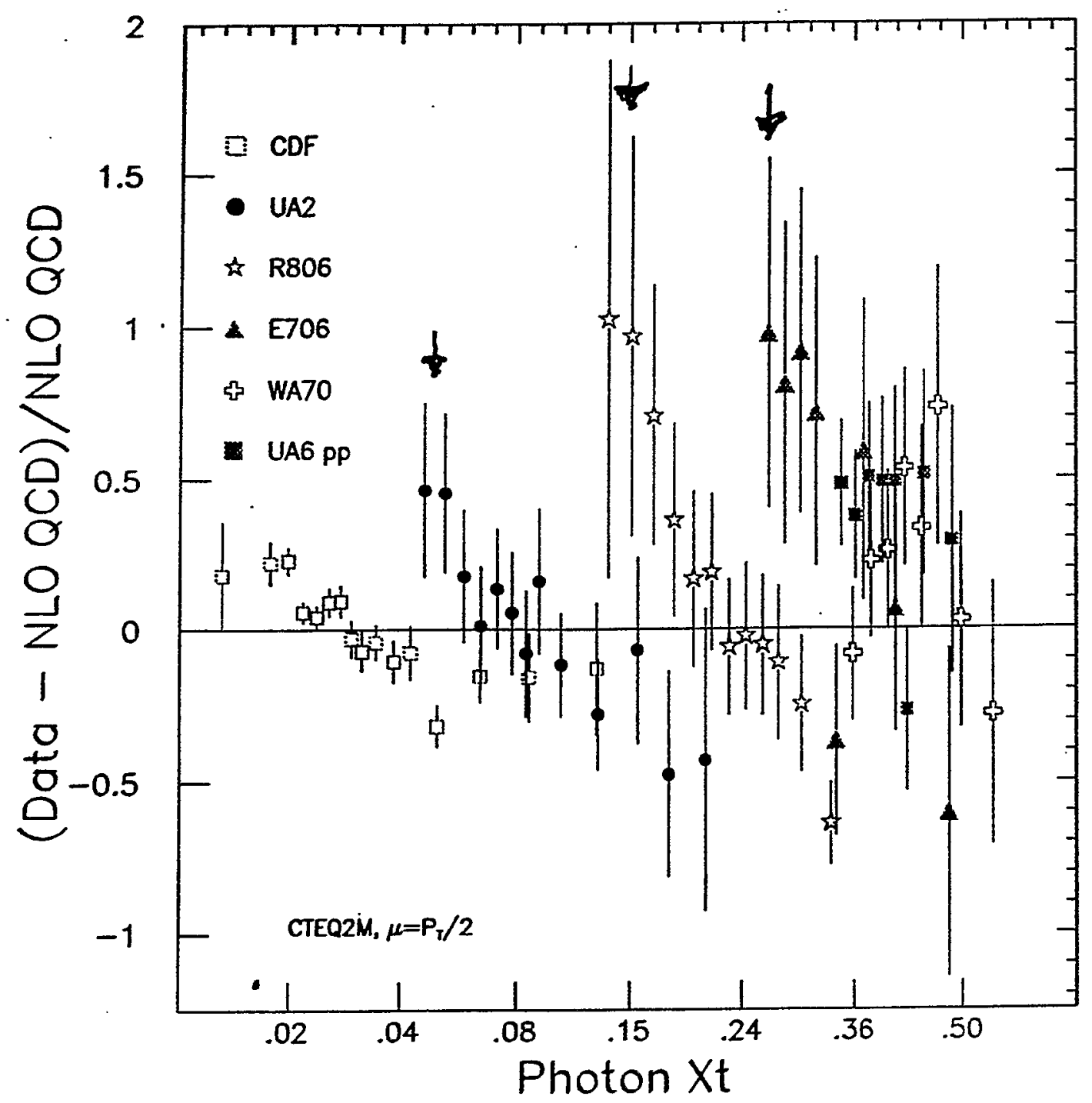

Figure 4: Compilation of direct photon experiments compared to NLO QCD predictions using CTEQ2M parton distributions. (see text) C peaks $^{3}$ at different $\sqrt{s} \rightarrow\left\{\begin{array}{l}\text { different } x_{t} \sim \frac{2 P_{t}}{\sqrt{s}} \\ \text { differ es }\end{array}\right.$ $\rightarrow$ not Coverall shape of $G(x)$.

62

$\sigma$ 


$$
\begin{gathered}
\text { E706 fixes CTEQ }{ }^{2} \text { wl } k_{T} \\
\text { smeaning: E706 Statss report } \\
23 \text { May } 97
\end{gathered}
$$

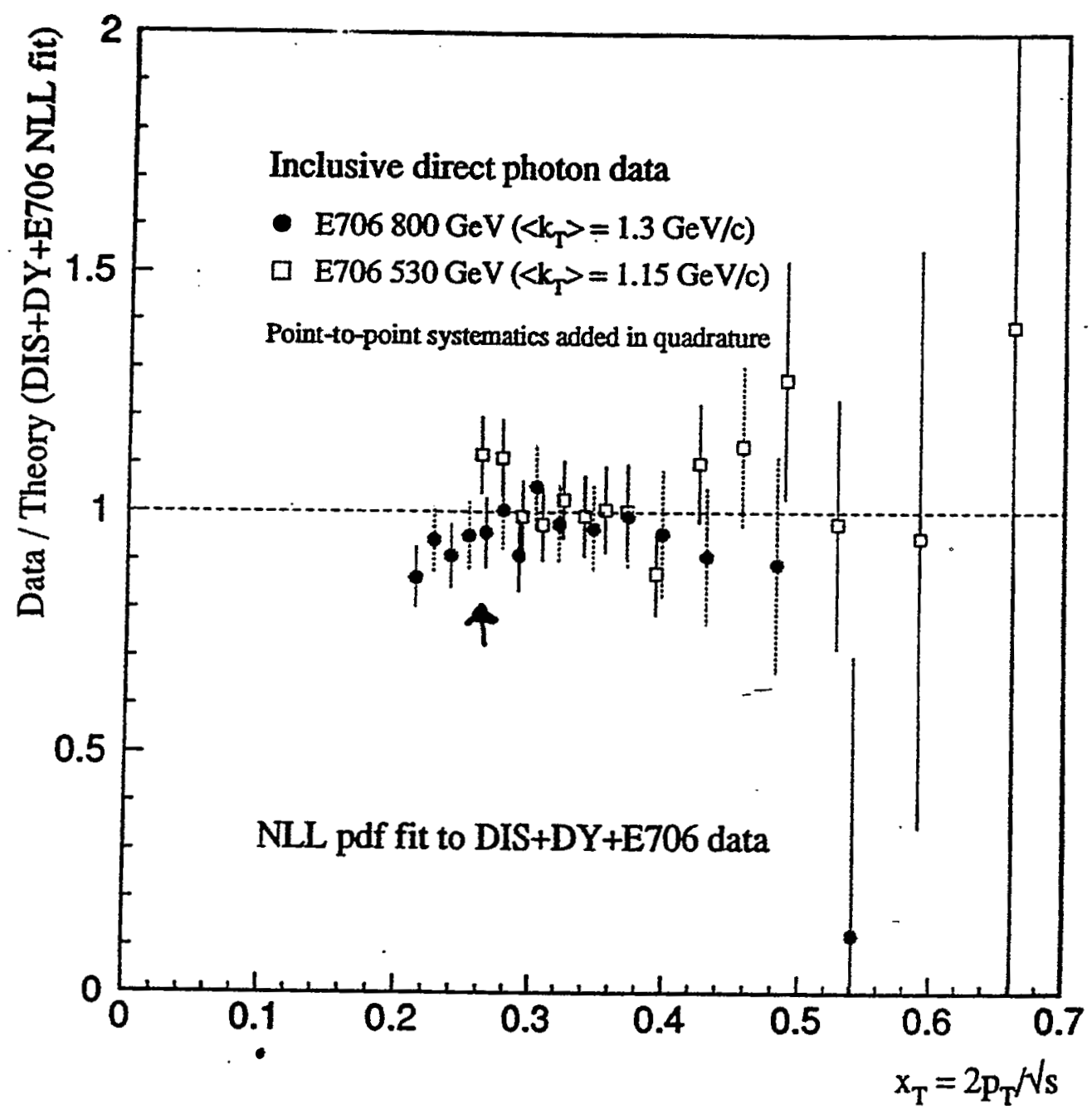

Figure 27: Ratio of our measured direct photon cross sections as a function of $x_{T} \equiv 2 p_{T} / \sqrt{s}$ for incident 530 and $800 \mathrm{GeV} / c$ proton beams to the cross section resulting from a NLL calculation using the gluon determined by fitting data from DIS, Drell-Yan, and the E706 direct photon cross sections. 
Can PT help account for these effects? Yes, take...

A closer look at higher orders: ' hidden logs of $k_{T}$

At NLC: soft glue in $\hat{\sigma}_{a g+\gamma x}$

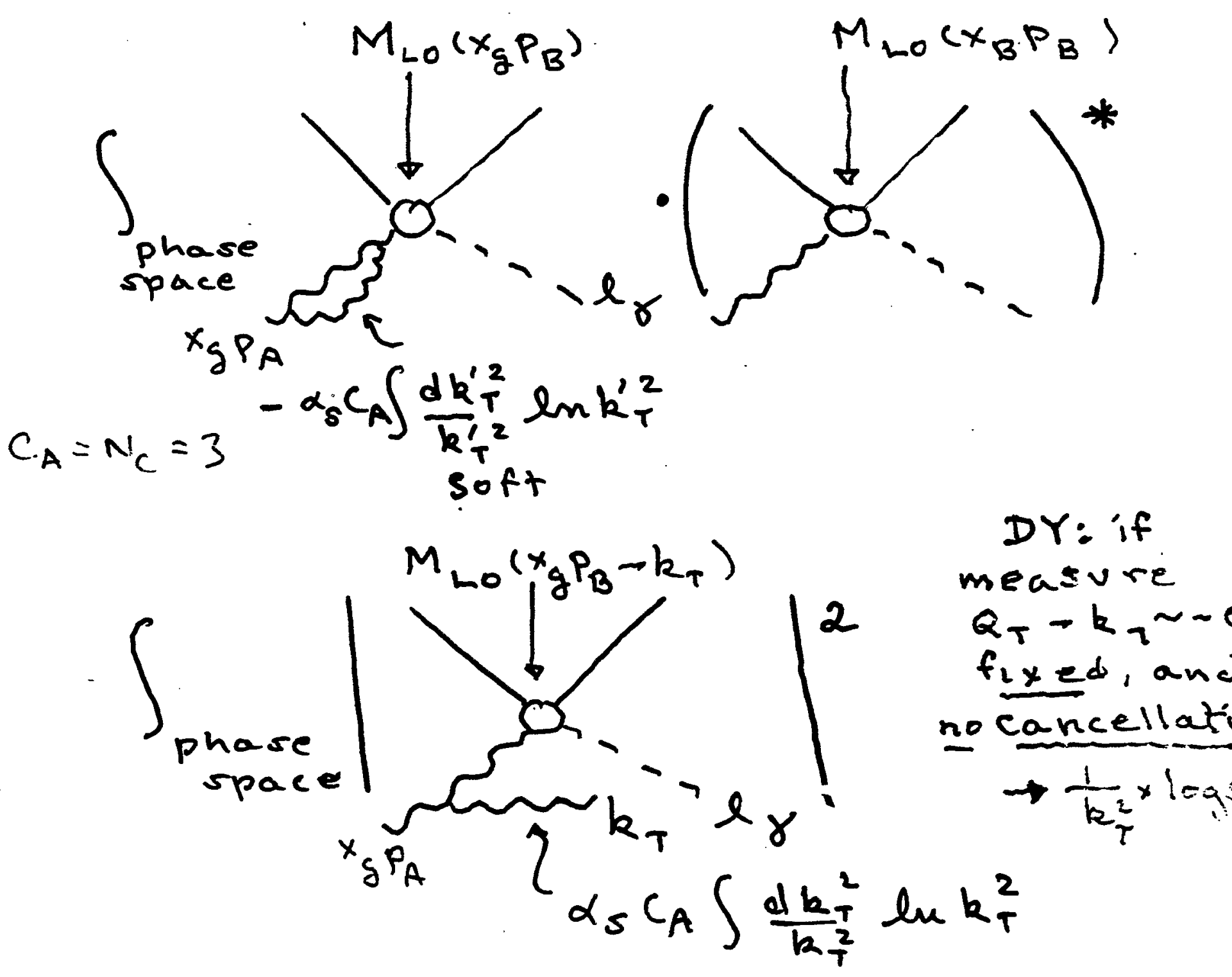

sum:

$$
\begin{aligned}
& -x_{S} C_{A}\left|M_{L 0}\left(x_{g} P_{A}\right)\right|^{2} \int \frac{d k_{T}^{\prime 2}}{k_{T}^{\prime 2}} \ln k_{T}^{\prime 2} \quad \begin{array}{l}
\text { cancellation } \\
\text { of diverge } \\
\text { at } k_{T} \rightarrow 0
\end{array} \\
& { }_{64}+\alpha_{S} c_{A} \int \frac{d k_{T}^{2}}{k_{T}^{2}} l_{M} k_{T}^{2} \mid M_{L_{0}}\left(x_{C} P_{A}-k_{\ldots} . .\right)
\end{aligned}
$$


Schematic results:

presumed $e x p o n e n t$

$\cong S(b, a)=$ perturbation

theory (PT)

+ non-perturbative (NP)

PT: sums $\ln ^{n} k^{2}, 1 Q^{2}$

cutoff in $I R$ ( $b_{0}$ above) 'parton shower'

$N P$ : induced by $P T$

also $b_{0}$-dependent

Gaussian smearing!$$
e^{-b^{2} \ln Q F_{2}+b^{2} F_{1}}
$$$$
e^{-9_{r}^{2} / 4\left(F_{2} \ln Q+F_{1}\right)}
$$

- Fourier transform

Q-dependent width

$$
\left\langle q_{T}^{2}\right\rangle \sim F_{2} \ln Q+F_{1}
$$

+ corrections

Full result: Convolution of resumed PT, NP with $L O+$ modified N LC $+\cdots$

Gaussian, dominates at low re and grows with $P_{T}$

65

16 


\section{Summary of the Discussion Session}

During the workshop, the scope of the work to be done before and at the next meeting, which is scheduled in March 1999, has been discussed. We agreed upon the need to provide a standard event generator for the RHIC spin program. The coming meeting will summarize the progress made until then in the various aspects with respect to which further improvements of the event generator is necessary. Here is the summary of our discussion for the direction of our work.

Polarized Generator vs Unpolarized One with Asymmetry Weights There was a comparison between results using the fully polarized event generator, which means to utilize polarized parton distribution and spin dependent cross sections and the results using an unpolarized event generator an accumulating events with the weights of the spin asymmetries in the polarized structure functions and cross sections. It was agreed that there is no significant difference in the results, but more detailed comparison is to be done. The fully polarized event generator is advantageous in the rigorous description of reactions. The latter method is advantageous not only to save on the CPU time required to obtain a certain precision, but also to update the event generator. Basically, no improvements in the polarization independent part are necessary so that we can concentrate on the improvements in the polarization dependent part of the program. Oliver Martin will perform more detailed comparisons of both methods. Les Bland and Naohito Saito will work together to combine two local versions of the program to calculate asymmetries in the structure functions and cross sections with the help of the Regensburg group.

\section{Implementation of new processes}

Since the polarized RHIC will open a completely new domain for spin physics, we expect to unveil new physics. Direct measurements for parity violating signals are a really powerful tool to search for implications of such new physics. Jean-Marc Virey and his colleague agreed to work on the implementation of such new processes into the event generator.

Heavy quark production in both bound and unbound states are expected to be very sensitive to the gluon distributions in the proton. Therefore, the spin asymmetries for these processes are expected to be a good probe 
for gluon polarization in the polarized protons. However the production mechanism of quarkonia is not well understood yet, and at least color singlet production which is currently included in the event generator is known to underestimate the production cross sections. It is now widely believed that inclusion of color octet states in the production is necessary to understand the cross section in the high energy domain. The color octet model is already included in a modified version of PYTHIA. Its asymmetry part is needed for our use. The TITech group have done relevant work recently, which should be included in the event generator.

As for the unbound heavy quark production, a leading order calculation of spin asymmetry is available. However, they mentioned the possibility of drastic cancelation of asymmetry due to large next-to-leading order contributions. There is no direct calculation available yet, and we are to contact some specialists to get a feeling for the relevance of these cancelations. We will decide on the basis of the results whether and how we should include such effects into the event generator.

\section{Implementation of new polarized parton distributions}

The primary goal of the RHIC spin program is to measure spin structure functions. It becomes "a must" for the event generator to provide a quick test of models for these structure functions. In the pre-experiment stage, a library of models on polarized parton distributions would be useful. The TITech group is going to take care of this part.

\section{Polarization Effects in the Fragmentation Process}

The spin effects in the fragmentation process is a very attractive area of research especially in the context of the measurements of transversity distributions in the proton. These effects are based on the partonic level asymmetry in spin transfer, $a_{T T}^{i f}$. It is, however, recognized as challenging task to implement such effects in the event generator, since there is no established model to describe the polarization effects in the fragmentation processes. Therefore we decided to rather include this topic into our long range plan.

\section{Comparison of Event Generator with NLO calculations}

Usually event generator contains only leading order cross sections. However it also includes initial and final state radiation. It thus simulates some next-to-leading order diagrams. To perform a fully consistent analysis of either structure functions or production mechanism, a good agreement be- 
tween the results of event generator runs and analytic calculations (at nextto-leading order) is crucial. As a starting point, we will compare the results of calculations using the event generator, analytic calculations, and most importantly experimental data. This work will be done by Lionel Gordon and experimentalists.

\section{Intrinsic Transverse Momentum of Partons}

Transverse momentum, $p_{T}$ of the final state particle is one of the most important variables in hadronic collisions. However, the $p_{T}$ will be highly affected by the intrinsic transverse momentum, $k_{T}$ of the partons in the initial state. It will be extremely difficult to reconstruct partonic kinematics without precise knowledge on the size of $k_{T}$. Actually the size of $k_{T}$ is one of the dominant source of the uncertainty in the gluon distribution as determined from prompt photon data.

Fortunately the size of $k_{T}$ can be constrained from experiments by looking at back-to-back correlations in particle production. Currently available experimental results can be explained with soft gluon emission, which has energy dependent transverse momentum distribution. Oliver Martin will implement the resulting model into the event generator.

\section{Comparison of PYTHIA, HERWIG and ISAJET}

Although these three major event generators started from different basis, their results are getting closer with every new revision. However, the difference in the fragmentation sensitive observables such as particle correlations in the final states is still visible. Therefore a careful comparison of these generators is necessary.

The results of all these efforts will be presented in the meeting next March, and a write-up will be produced to summarize the status. Obviously efforts to improve the event generator should be an on-going project, and the March meeting will only mark the initial milestone of our work. We hope very much to contribute by it to the success of the spin program at RHIC.

$\begin{array}{lll}\text { Andreas Schäfer } & \text { Naohito Saito } & \text { Les Bland } \\ \text { Stefano Frixione } & \text { Lionel E. Gordon } & \text { Basim Kamal } \\ \text { Oliver Martin } & \text { Martin Maul } & \text { Frank Paige } \\ \text { Toshi-Aki Shibata } & \text { George Sterman } & \text { Jean-Marc Virey } \\ \text { Werner Vogelsang } & & \end{array}$




\section{Workshop on Event Generator for RHIC SPIN Physics SEPTEMBER 21-23, 1998 \\ ORGANIZERS: NAOHITO SATTO AND ANDREAS SCHAFER}

\section{LIST OF PARTICIPANTS}

\begin{tabular}{|c|c|c|}
\hline NAME & Affiliation and Address & E-MAILADDRESS \\
\hline Les Bland & $\begin{array}{l}\text { IUCF } \\
2401 \text { Milo B. Sampson Lane } \\
\text { Bloomington, IN } 47408\end{array}$ & bland@iucf.indiana.edu \\
\hline Claudio Coriano & $\begin{array}{l}\text { Jefferson Laboratory } \\
\text { Cebaf Theory } \\
12000 \text { Jefferson Avenue } \\
\text { Newport News, VA } 23606 \text { USA }\end{array}$ & coriano@jlab.org \\
\hline Geary Eppley & $\begin{array}{l}\text { Rice University } \\
\text { Bonner Lab - MS } 315 \\
6100 \text { main Street } \\
\text { Houston, TX 77005 USA }\end{array}$ & eppley@physics.rice.edu \\
\hline Lionel Gordon & $\begin{array}{l}\text { Thomas Jefferson National Accelerator } \\
\text { Facility } \\
12000 \text { Jefferson Avenue } \\
\text { Newport News, VA } 23606\end{array}$ & gordon@hep.anl.gov \\
\hline Yuji Goto & $\begin{array}{l}\text { RIKEN } \\
\text { Brookhaven National Laboratory } \\
\text { Physics Department, Bldg. } 902 \\
\text { P.O. Box } 5000 \\
\text { Upton, NY } 11973-5000 \text { USA }\end{array}$ & goto@bnl.gov \\
\hline Tim Hallman & $\begin{array}{l}\text { Brookhaven National Laboratory } \\
\text { Physics Department, Bldg.510A } \\
\text { P.O. Box 5000 } \\
\text { Upton, NY } 11973-5000 \text { USA }\end{array}$ & hallman@bnl.gov \\
\hline Naoki Hayashi & $\begin{array}{l}\text { RIKEN } \\
\text { Brookhaven National Laboratory } \\
\text { AGS Department, Bldg. } 911 \\
\text { P.O. Box } 5000 \\
\text { Upton, NY } 11973-5000 \text { USA }\end{array}$ & hayashi@bnl.gov \\
\hline Bob Jaffe & $\begin{array}{l}\text { Center for Theoretical Physics \& Labora- } \\
\text { tory for Nuclear Science } \\
\text { Dept. of Physics, Room 6-311 } \\
\text { Massachusetts Inst. of Tech. } \\
\text { Cambridge, MA 02139 }\end{array}$ & jaffe@mitlns.mit.edu \\
\hline Basim Kamal & $\begin{array}{l}\text { Physics Department, Bldg. 510A } \\
\text { Brookhaven National Laboratory } \\
\text { P.O. Box } 5000 \\
\text { Upton, NY } 11973-5000 \text { USA }\end{array}$ & kamal@wind.phy.bnl.gov \\
\hline Kazu Kurita & $\begin{array}{l}\text { RIKEN/RBRC } \\
\text { Brookhaven National Laboratory } \\
\text { P.O. Box } 5000 \\
\text { Upton, NY } 11973-5000 \text { USA }\end{array}$ & kurita@bnl.gov \\
\hline
\end{tabular}




\section{Workshop On EVENT GeNerator for RHIC SPIN PhySICS SEPTEMBER 21-23, 1998 \\ ORGANIZERS: NAOHITO SATTO AND ANDREAS SCHAFER}

\section{LIST OF PARTICIPANTS}

\begin{tabular}{|c|c|c|}
\hline $\begin{array}{l}\text { NAME } \\
\text { Yajun Mao }\end{array}$ & $\begin{array}{l}\text { Affiliation and Address } \\
\text { Brookhaven National Labaoratory } \\
\text { Bldg. } 510 \mathrm{C} \\
\text { P.O. Box } 5000 \\
\text { Upton, NY } 11973-5000 \text { USA }\end{array}$ & $\begin{array}{l}\text { E-MAIL ADDRESS } \\
\text { mao@riksg01.rhic.bnl.gov }\end{array}$ \\
\hline Oliver Martin & $\begin{array}{l}\text { Lehrstuhl Prof. Schaefer } \\
\text { Institut fuer Theoretische Physik I } \\
\text { Universitaetsstr. } 31 \\
\text { Universitaet Regensburg } \\
93040 \text { Regensburg, Germany }\end{array}$ & oliver.martin@physik.uni-regensburg.de \\
\hline Martin Maul & $\begin{array}{l}\text { Nordisk Institute for Theoretical Physics } \\
\text { Blegdamsvej } 17 \\
\text { DK-2100 Copenhagen, Denmark }\end{array}$ & martin.maul@physik.uni-regensburg.de \\
\hline Frank Paige & $\begin{array}{l}\text { Brookhaven National Labaoratory } \\
\text { Physics Department, Bldg. 510A } \\
\text { P.O. Box } 5000 \\
\text { Upton, NY } 11973-5000 \text { USA }\end{array}$ & paige@bnl.gov \\
\hline Naohito Saito & $\begin{array}{l}\text { RIKEN/RBRC } \\
\text { Physics Department, Bldg. 510C } \\
\text { Brookhaven National Laboratory } \\
\text { P.O. Box } 5000 \\
\text { Upton, NY } 11973-5000 \text { USA }\end{array}$ & saito@bnl.gov \\
\hline Hiroki Sato & $\begin{array}{l}\text { Brookhaven National Laboratory } \\
\text { Bldg.902C } \\
\text { P.O. Box } 5000 \\
\text { Upton, NY } 11973-5000 \text { USA }\end{array}$ & satohiro@pn.scphys.kyoto-u.ac.jp \\
\hline Andreas Schaefer & $\begin{array}{l}\text { Institut für Theoretische Physik } \\
\text { Universität Regensburg } \\
\text { D-93040 Regensburg, Germany }\end{array}$ & andreas1.schaefer@physik.uni-regensburg.de \\
\hline Toshi-Aki Shibata & $\begin{array}{l}\text { Faculty of Science } \\
\text { Tokyo Institute of Technology } \\
\text { Ookayama, Meguro-ku } \\
\text { Tokyo 152, Japan }\end{array}$ & shibata@nucl.phys.titech.ac.jp \\
\hline George Sterman & $\begin{array}{l}\text { Institute for Theoretical Physics } \\
\text { SUNY, Stony Brook } \\
\text { Srony Brook, NY 11794-3840 }\end{array}$ & sterman@nuclear.physics.sunysb.edu \\
\hline Mike Tannenbaum & $\begin{array}{l}\text { Brookhaven National Laboratory } \\
\text { Physics Department, Bldg. 510C } \\
\text { P.O. Box } 5000 \\
\text { Upton, NY } 11973-5000 \text { USA }\end{array}$ & mjt@bnl.gov \\
\hline Christos Velissaris & $\begin{array}{l}\text { Brookhaven National Laboratory } \\
\text { Building } 902 C \\
\text { P.O. Box } 5000 \\
\text { Upton, NY } 11973-5000 \text { USA }\end{array}$ & christos@rcf.rhic.bnl.gov \\
\hline
\end{tabular}




\title{
RIKEN BNL RESEARCH CENTER
}

\author{
Workshop on Event GeNerator for RHIC SPIN Physics \\ SEPTEMBER 21-23, 1998 \\ Physics DePaRTMENT - RoOM 2-160 \\ ORGANIZERS: NAOHTO SAITO AND ANDREAS SCHAEFER
}

\section{AGENDA}

\section{Monday Morning, September 21}

Chair: Toshi-Aki Shibata

\author{
09:00 Workshop Introduction from Organizing Committee - Naohito Saito \\ 09:10 RHIC Spin Physics - Andreas Schaefer \\ 10:10 Event Generator and RHIC Spin Experiments - Naohito Saito \\ 10:45 Coffee Break \\ 11:15 SPHINX - A Polarized Event Generator: Basics and Future Developments \\ of MV Event Generators - Oliver Martin \\ 12:15 Lunch
}

\section{Monday Afternoon, September 21 \\ Chair: Andreas Schaefer}

14:00 Event Generator: Unpolarized Case - Frank Paige

15:00 NLO Calculation on Prompt Photon Production in Polarized pp Collisions - Lionel Gordon

16:00 Coffee Break

16:15 Event Generators for Special Processes: Examples for ep Collisions - Martin Maul

\section{Tuesday Morning, September 22}

Chair: Naohito Saito

09:00 STAR Spin Physics - Leslie Bland

10:00 Spin Discussion at Orange Room

11:00 Coffee Break

11:15 PHENIX Spin Physics - Naoki Hayashi

12:15 Lunch 
Tuesday Afternoon, September 22

Chair: Frank Paige

16:30 Polarized Parton Distributions and Event Generator — Toshi-Aki Shibata

\section{Wednesday Morning, September 23}

Chair: Naohito Saito

09:00 Resummation and intrinsic p T - George Sterman

10:00 Discussion of Future Work

12:00 Adjourn 

